\title{
Keys to the avian malaria parasites
}

CrossMark

\author{
Gediminas Valkiūnas* (iD and Tatjana A. lezhova
}

\begin{abstract}
Background: Malaria parasites (genus Plasmodium) are widespread in birds. These pathogens cause pathology of blood and various organs, often resulting in severe avian malaria. Numerous recent studies have reported DNA sequences of avian malaria parasites, indicating rich genetic diversity and the possible existence of many undescribed species. However, the majority of reported Plasmodium lineages remain unidentified to species level, and molecular characterization is unavailable for the majority of described Plasmodium parasites. During the past 15 years, numerous new Plasmodium species have been described. However, keys for their identification are unavailable or incomplete. Identification of avian malaria parasites remains a difficult task even for experts, and this precludes development of avian malariology, particularly in wildlife. Here, keys for avian malaria parasites have been developed as a baseline for assisting academic and veterinary medicine researchers in identification of these pathogens. The main obstacles and future research priorities have been defined in the taxonomy of avian Plasmodium species.
\end{abstract}

Methods: The data were considered from published articles and type and voucher material, which was accessed in museums in Europe, the USA and Australia. Blood films containing various blood stages of the majority of described species were examined and used for the development of dichotomous keys for avian Plasmodium species.

Results: In all, 164 published articles were included in this review. Blood stages of avian Plasmodium parasites belonging to subgenera Haemamoeba, Giovannolaia, Novyella, Bennettinia and Huffia were analysed and compared. Illustrated keys for identification of subgenera and species of these parasites were developed. Lists of invalid and synonymous Plasmodium parasite names as well as names of doubtful identity were composed.

Conclusion: This study shows that 55 described species of avian Plasmodium can be readily identified using morphological features of their blood stages. These were incorporated in the keys. Numerous synonymous names of Plasmodium species and also the names belonging to the category species inquirenda exist, and they can be used as reserves for future taxonomy studies. Molecular markers are unavailable for $58 \%$ of described Plasmodium parasites, raising a task for the current avian malaria researchers to fill up this gap.

Keywords: Avian malaria, Key to species, Plasmodium, Species inquirenda, Synonym, Avian Plasmodium taxonomy

\section{Background}

Malaria parasites of the genus Plasmodium (Haemosporida, Plasmodiidae) inhabit all major groups of terrestrial vertebrates. Avian malaria parasites is a peculiar group among them, particularly due to the ability of numerous species to develop and complete life cycles in numerous bird species belonging to different families and even orders [1-7]. The same is true for invertebrate hosts (vectors) of these parasites $[8,9]$. Many species of avian Plasmodium use Culicidae mosquitoes belonging to different

*Correspondence: gedvalk@ekoi.lt

Nature Research Centre, Akademijos 2, 08412 Vilnius 2100, Lithuania genera (Culex, Coquillettidia, Aedes, Mansonia, Culisetta, Anopheles, Psorophora) for completing sporogony and transmission [1, 8-11]. This is not the case in mammalian malaria parasites whose are transmitted mostly by Anopheles species [1, 12-14]. Furthermore, sporogony of many avian Plasmodium parasites is completed relatively fast in susceptible vectors at relatively low temperatures $[1,8,15,16]$. These features likely contributed to the global distribution of some avian malaria infections, which are actively transmitted in countries with warm and cold climates, including regions close to the Polar Circles [6, 17-19]. 
Life cycles of avian malaria parasites are similar in their basic features to those of human and other mammal Plasmodium species [1, 2, 8, 13, 14, 20]. Malaria parasites are obligate heteroxenous protists, with merogony in cells of fixed tissues and also blood cells. Gametogony occurs in red blood cells, and sexual process and sporogony are completed in Culicidae mosquitoes. However, the life cycles of avian Plasmodium species differ from those of the parasites of mammals, particularly due to their relatively low host specificity and marked variation in patterns of development in avian hosts and vectors. For example, Plasmodium (Haemamoeba) relictum infects and completes its life cycle in birds belonging to over 300 species and 11 orders, and Plasmodium (Huffia) elongatum, Plasmodium (Novyella) vaughani and many other species also have a broad range of avian hosts [6, 8, 21-23]. Erythrocytic merozoites of many avian malaria parasites can induce secondary tissue merogony in birds $[24,25]$. The exo-erythrocytic merogony occurs in cells of the reticuloendothelial and haemopoietic systems, but has not been reported in hepatocytes [2, 4, 8, 23, 26]. Pedunculated oocysts were discovered in Plasmodium (Bennettinia) juxtanucleare; these oocysts possess leglike outgrowths which attach the oocysts to the mosquito midgut wall [27]. These and some other features are not characteristics of malaria parasites of mammals, and this is reflected in genetic differences between these groups of parasites and their different position in molecular phylogenies [28-33].

Malaria, the disease caused by parasites of the genus Plasmodium, has traditionally been viewed as a disease of the blood and blood forming tissues of vertebrate hosts, with exo-erythrocytic stages of development causing little or no pathology $[1,13,14,34]$. While available evidence still supports this view for the primate and rodent malarial parasites, there is increasing evidence that the pathogenicity of tissue stages of avian species of Plasmodium has been significantly underestimated [25]. Even more, avian malaria is often a more severe disease than human malaria. There is recent experimental evidence of unexpected pathology associated with obstructive development of secondary exo-erythrocytic stages of Plasmodium in brain capillaries that can lead to ischaemia and rapid death in birds that have very low intensity parasitaemias during chronic stage of infection [24, 25, 35]. Importantly, the severity of disease caused by a given lineage of Plasmodium often varies markedly in different species of avian hosts, from absence of any clinical symptoms to high mortality [4, 17, 19, 36-41].

Because of broad vertebrate host specificity, the same Plasmodium species can infect distantly related birds. In other words, vertebrate host identity cannot be used as a taxonomic feature during identification of avian malaria parasites [1, 12, 42]. This raises questions about parasite species identification if the same pathogen is found in unusual avian hosts. Molecular characterization is helpful in diagnosis of malaria infections, and has been developed for detection of some avian Plasmodium species $[21,40]$. Molecular markers are essential in diagnosis and identification of exo-erythrocytic and vector stages, which cannot be identified using morphological features $[11,43,44]$. However, molecular diagnostics using general primers (the main diagnostic tool currently used in wildlife malariology) is often insensitive in distinguishing of avian Plasmodium spp. co-infections, which are common and even predominate in many bird populations [45-48]. Specific molecular markers for the majority of avian Plasmodium species have not been developed, and currently are difficult to develop due to significant genetic diversity of malaria parasites, which remain undescribed in wildlife. Morphological identification using microscopic examination of blood films remains important in malaria diagnostics in the wild, and is particularly valuable if it is applied in parallel with polymerase chain reaction (PCR)-based diagnostic tools [5, 30, 49, 50].

During the past 15 years, numerous avian Plasmodium parasites were named and described using morphological features of their blood stages [49, 51-59]. However, molecular markers for parasite detection were developed in a handful of these descriptions. The keys that are available for identification of avian Plasmodium species [8], should be reworked in the light of the newly available information.

The main aim of this review is to develop easy-to-use keys for identification of avian malaria parasites using morphological features of their blood stages as a baseline for assisting academic and veterinary medicine researchers in identification of these pathogens. Lists of synonymous names of Plasmodium species as well as invalid species names were updated and compiled. The Plasmodium parasite names of unknown taxonomic position (incertae sedis) and the species of doubtful identity requiring further investigation (species inquirenda) were specified as well. The information about useful molecular markers, which can be used for described Plasmodium species detection and comparison was also summarized. This review might be helpful for wildlife malaria and veterinary medicine researchers aiming identification of avian malaria infections.

\section{Methods}

Full-length papers with descriptions of new Plasmodium species published in peer-reviewed journals were considered. In all, 164 articles were reviewed, and 152 papers containing most representative information about 
taxonomy of these parasites were incorporated in the References.

Type and voucher preparation as well as images of blood stages of avian Plasmodium parasites were obtained from the collections of Natural History Museum (London, UK), International Reference Centre for Avian Haematozoa (Queensland Museum, Quensland, Australia), the US National Parasite Collection (National Museum of Natural History, Washington DC, USA), Muséum National d'Histoire Naturelle (Paris, France), Grupo de Estudio Relación Parásito Hospedero, Universidad Nacional de Colombia (Bogotá, Colombia) and Nature Research Centre (Vilnius, Lithuania). All accessed preparations were studied. An Olympus BX61 light microscope (Olympus, Tokyo, Japan) equipped with an Olympus DP70 digital camera and imaging software AnalySIS FIVE (Olympus Soft Imaging Solution GmbH, Münster, Germany) was used to examine preparations and prepare illustrations.

A method of dichotomous key was applied for identification of Plasmodium species. This tool consists of steps divided it two alternative parts, which allow to determine the identity of a specimen due to a series of choices that lead the user to the correct name of a given specimen. The most difficult choices, which do not exclude ambiguity, were accompanied with references to the corresponding pictures, which illustrate meaning of the text information. This simplifies the comparison of diagnostic features used in the keys. All parasite names in the keys are accompanied with references to the original parasite descriptions and (or) reviews containing description and (or) illustrations of corresponding species.

\section{Results}

Birds are often infected with different blood parasites belonging to same and different genera in the wild, and various combinations of different parasite co-infections often occur in same individual hosts. Haemosporidians (order Haemosporida) develop intracellularly, and they should be distinguished from other eukaryotic intracellular infections before identification of the parasite species identity. Haemosporidians can be readily distinguished from all other intracellular protists (species of Babesia, Isospora, Lankesterella, Haemogrerina, Hepatozoon, Toxoplasma) due to one particularly readily distinguishable feature. Mainly, gametocytes of all haemosporidians are characterized by sexually dimorphic features, which are readily distinguishable under the light microscope. Haemosporidian macrogametocytes possess compact nuclei and bluish-stained cytoplasm, and the microgametocyte nuclei are diffuse and the cytoplasm stains paler than in macrogametocytes (compare Fig. 1a, h with b, i). Some variation occurs in the size of nuclei and in the staining of the cytoplasm in different haemosporidian species. While, this also depends on staining protocols, macroand microgametocytes can be readily distinguished in each haemosporidian species. This is not the case in other intracellular protists, whose gamonts and other intracellular blood stages do not show sexually dimorphic features and all look similar under the light microscope (Fig. $1 \mathrm{j}-1$ ).

Based on current taxonomy, four families of haemosporidians can be recognized. These are Plasmodiidae, Haemoproteidae, Leucocytozoidae and Garniidae [1, 4, $8,30,60,61]$. Malaria parasites are classified in the family Plasmodiidae, which contains one genus Plasmodium. When haemosporidians are found in blood films, Plasmodium parasites should be distinguished from species of related haemosporidians belonging to the families Garniidae, Haemoproteidae and Leucocytozoidae. The main distinctive features of parasites belonging to these families are summarized in Table 1.

Blood stages of species of Plasmodium are particularly similar to those of relatively rare haemosporidian parasites of the genera Fallisia and Garnia of the family Garniidae [8, 60-62]. Parasites of these three genera produce gametocytes and meronts (=schizonts) in blood cells (Fig. 1a-f). However, species of Plasmodium do not digest haemoglobin completely and accumulate residual pigment granules (hemozoin), which are refractory and readily visible in blood stages under light microscope (Fig. 1a-c). This is not true of species belonging to the genera Fallisia and Garnia or other garniids, which digest haemoglobin completely when they inhabit red blood cells and do not possess pigment granules in their blood stages (Fig. 1d-f).

When malaria parasites of the Plasmodium genus are reported in blood films, the next step is to distinguish subgenera of this genus. The main characteristics of different subgenera are summarized in Table 2.

When the subgenus of a malaria parasite has been identified, the next step is the species identification using the keys to species (Tables 3, 4, 5, 6).

\section{Discussion}

There are three main groups of obstacles, which a researcher usually faces during morphological identification of malaria parasites using microscopic examination of blood samples collected in the field. First, the quality of microscopic preparations is essential for correct parasite identification, but often is insufficient due to thick blood films or artefacts of their drying, fixation, staining or storage. This precludes visualization of some important features for species identification. It is essential to master these simple methods of traditional parasitology before sample collection, and this can be readily achieved 

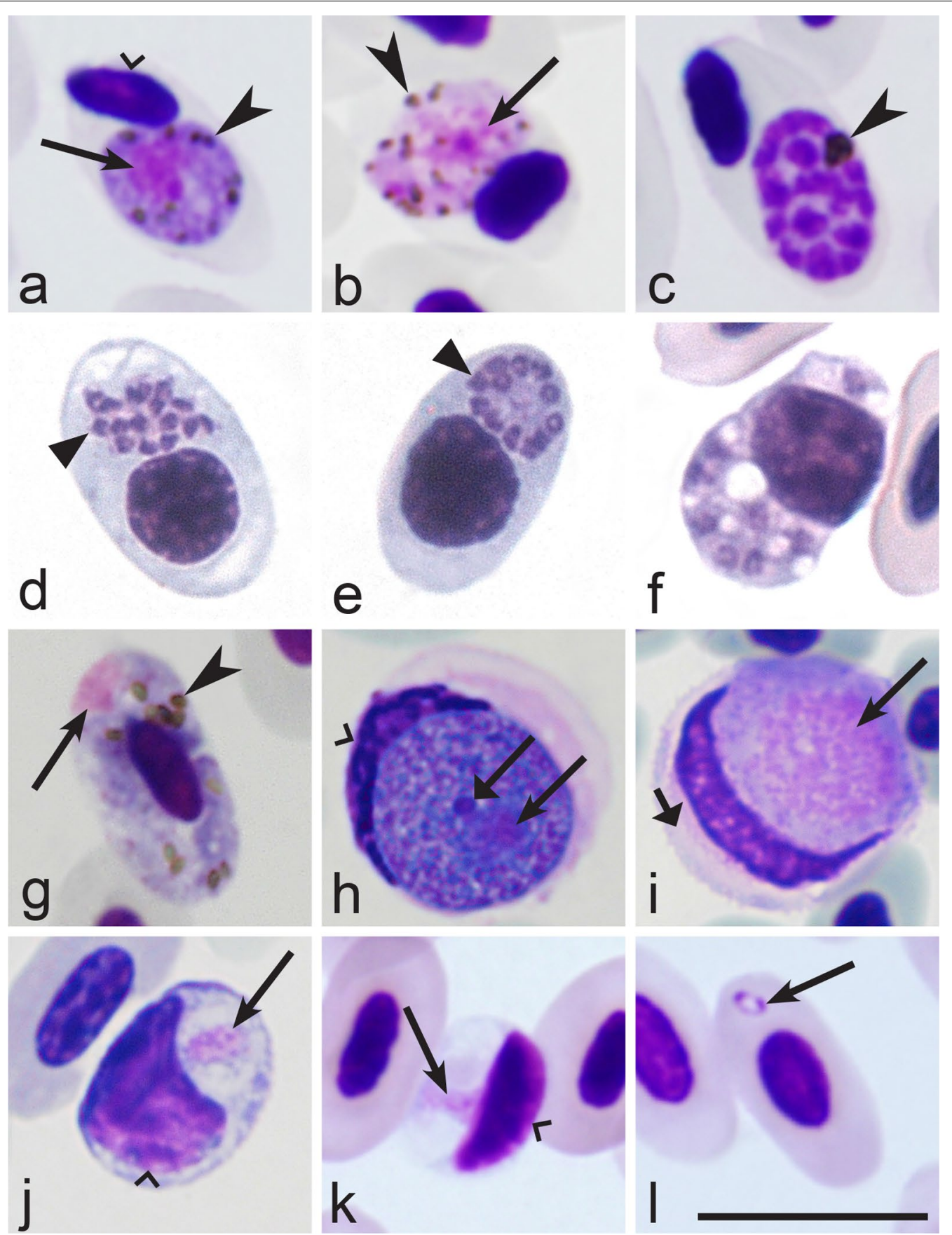

Fig. 1 Main morphological features of blood stages, which are used for identification of families of haemosporidian (Haemosporida) parasites $(\mathbf{a}-\mathbf{i})$. Mature gametocytes $(\mathbf{a}, \mathbf{b}, \mathbf{g}-\mathbf{i})$ and meronts $(\mathbf{c}-\mathbf{f})$ of Plasmodium $(\mathbf{a}-\mathbf{c})$, Garnia $(\mathbf{d}, \mathbf{e})$, Fallisia $(\mathbf{f})$, Haemoproteus $(\mathbf{g})$ and Leucocytozoon $(\mathbf{h}, \mathbf{i})$ parasites belonging to the families Plasmodiidae $(\mathbf{a}-\mathbf{c})$, Garniidae $(\mathbf{d}-\mathbf{f})$, Haemoproteidae $(\mathbf{g})$ and Leucocytozoidae $(\mathbf{h}$, i). Note presence of malarial pigment in species of Plasmodiidae $(\mathbf{a}-\mathbf{c})$ and Haemoproteidae $(\mathbf{g})$ and its absence in species of Garniidae $(\mathbf{d}-\mathbf{f})$ and Leucocytozoidae $(\mathbf{h}, \mathbf{i})$. Macrogametocytes $(\mathbf{a}, \mathbf{g}, \mathbf{h})$ and microgametocytes $(\mathbf{b}, \mathbf{i})$ are readily distinguishable due to presence of sexually dimorphic features. Common avian intracellular non-haemosporidian parasites $(\mathbf{j}-\mathbf{I})$ are shown for comparison with haemosporidians. These are Isospora (synonym Atoxoplasma) (j), Hepatozoon (k) and Babesia (I). Long simple arrows_-nuclei of parasites. Simple arrowhead — pigment granules. Triangle arrowheads_developing merozoites. Long simple wide arrow—nucleolus. Simple wide arrowheads—host cell nuclei. Short simple wide arrow—cytoplasm of host cell. Scale $\mathrm{bar}=10 \mu \mathrm{m}$. Explanations are given in the text 
Table 1 Key to families of haemosporidian parasites

\begin{tabular}{|c|c|}
\hline Step & Features and family \\
\hline $1(4)$ & Merogony takes place in blood cells (Fig. 1c-f) \\
\hline \multirow[t]{2}{*}{$2(3)$} & Malarial pigment (hemozoin) is present in blood stages (Fig. 1a-c) \\
\hline & 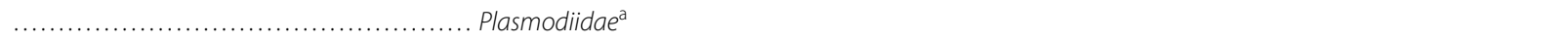 \\
\hline \multirow[t]{2}{*}{$3(2)$} & Malarial pigment (hemozoin) is absent from blood stages (Fig. 1d-f) \\
\hline & Garniidae \\
\hline $4(1)$ & Merogony (Fig. 1c-f) does not take place in blood cells. Only gametocytes (Fig. 1g-i) present in blood cells \\
\hline \multirow[t]{2}{*}{$5(6)$} & Malarial pigment (hemozoin) is present in blood stages (Fig. 1a, b, g) \\
\hline & 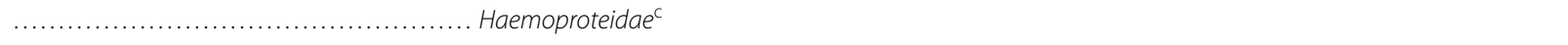 \\
\hline \multirow[t]{2}{*}{$6(5)$} & Malarial pigment (hemozoin) is absent from blood stages (Fig. 1h, i) \\
\hline & Leucocytozoidae \\
\hline \multicolumn{2}{|r|}{ Main taxonomic features of families of the haemosporidian parasites [8] } \\
\hline \multicolumn{2}{|r|}{$\begin{array}{l}\text { a Merogony takes place in cells of fixed tissues and blood cells of vertebrate hosts. Malarial pigment (hemozoin) is present in erythrocytic meronts and gametocytes. } \\
\text { Sexual process and sporogony of bird parasites take place in mosquitoes (Diptera: Culicidae) }\end{array}$} \\
\hline \multicolumn{2}{|r|}{ b Merogony takes place in cells of fixed tissues and blood cells of vertebrate hosts. Malarial pigment (hemozoin) is absent at all stages. Vectors are still unknown } \\
\hline \multicolumn{2}{|r|}{$\begin{array}{l}\text { c Merogony takes place in cells of fixed tissues of vertebrate hosts. No merogony occurs in blood cells. Malarial pigment (hemozoin) is present in gametocytes. Sexual } \\
\text { process and sporogony of bird parasites take place in louse flies (Hippoboscidae) and biting midges (Ceratopogonidae) }\end{array}$} \\
\hline \multicolumn{2}{|r|}{$\begin{array}{l}\text { d Merogony takes place in cells of fixed tissues of vertebrate hosts. No merogony occurs in blood cells. Malarial pigment (hemozoin) is absent at all stages. Sexual } \\
\text { process and sporogony take place in black flies (Simuliidae) and biting midges (Ceratopogonidae) }\end{array}$} \\
\hline
\end{tabular}

in each laboratory using available protocols $[1,8,63,64]$. Second, Plasmodium species parasitaemia is often light in natural infections in the wild. In other words, malaria parasites might be reported in blood films, but not all stages, which are needed for parasite species identification, are present. This might limit the use of the keys. Sampling of large number of birds (20-30 individuals) belonging to the same species at a study site is often helpful to detect relatively high parasitaemia of the same pathogen and to access the full range of blood stages allowing parasite species identification. Third, co-infections of Plasmodium species might occur, and requires some experience to distinguish between different pathogens $[45,48,56]$. These obstacles strengthen the need for the development of molecular characterization in avian malaria diagnostics, which is still only available for $44 \%$ of described parasite species, whose validity is obvious (Table 7). This is particularly timely for itemizing Plasmodium species phylogenies, which currently are based mainly on mitochondrial cytb gene sequences in avian malariology [5, 7, 23, 29, 33].

Molecular markers are sensitive for distinguishing different parasite species and their lineages, and they are essential for identification of cryptic Plasmodium species [35]. Molecular characterization is best developed for Novyella parasites (molecular markers are available for $59 \%$ of described species of this subgenus), and is weakest for Giovannolaia parasites (only two species or $12.5 \%$ of this subgenus have been characterized molecularly). Lack of molecular markers for many described malaria pathogens $[51,53,54,56,57,59,65]$ precludes biodiversity research on Plasmodium species and recognition of new malaria pathogens, for whose detection, detailed comparison with already described and genetically characterized parasites is needed. The development of molecular markers for diagnosis of disease agents is an important task of current avian malariology (Table 7).

This study shows that 55 described species of avian malaria parasites can be readily distinguished (Tables 3 , $4,5,6,7)$. Among them, 12, 16, 22, 4 and 1 species belong to subgenera Haemamoeba, Giovannolaia, Novyella, Huffia and Bennettinia, respectively. The great majority of described avian Plasmodium species were reported only in birds that live in tropical and subtropical countries or in Holarctic migrants wintering in the same regions, indicating that transmission of these pathogens occurs mainly in countries with warm climates. Those malaria parasites, which have adapted for transmission globally and have become cosmopolitan, are exceptions. Among these, Plasmodium relictum, Plasmodium elongatum, Plasmodium circumflexum, Plasmodium matutinum and Plasmodium vaughani should be mentioned first of all $[6,8,21,23,66-70]$. These are invasive infections, which are often virulent in non-adapted hosts, and they are worth particular attention in bird health.

Among described avian Plasmodium parasites, species of Novyella are particularly diverse (Table 5). They represent approximately $40 \%$ of all described avian malaria pathogens, and $78 \%$ of Plasmodium species, which were discovered during past 15 years. Novyella parasites are mainly pathogens of birds in countries of tropical and subtropical regions (Table 5). The Holarctic migrating 
Table 2 Key to subgenera of Plasmodium parasites of birds

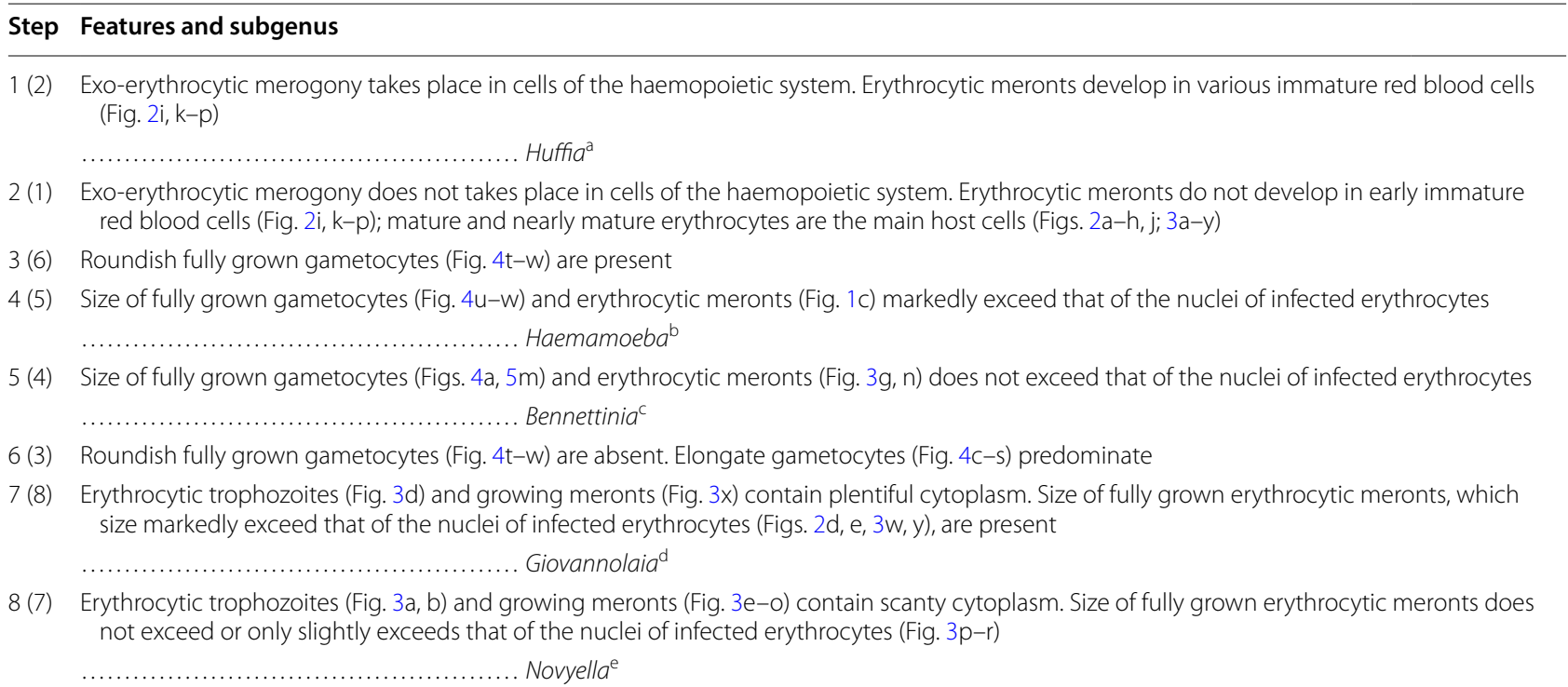

Main taxonomic characters of subgenera of avian malaria parasites [8]

a Exo-erythrocytic merogony takes place in cells of the haemopoietic system. Erythrocytic trophozoites and growing meronts (Fig. 2l-p) contain plentiful cytoplasm. Erythrocytic meronts develop in various immature red blood cells (Plasmodium huffi probably is an exception, but this needs confirmation). Fully grown erythrocytic meronts and gametocytes are variable both in form and size; elongate, roundish and irregularly shaped parasites might occur. Pedunculated oocysts are absent

${ }^{b}$ Exo-erythrocytic merogony takes place in cells of the reticuloendothelial system. Erythrocytic trophozoites (Fig. 3d) and growing meronts (Fig. 2a, b) contain plentiful cytoplasm. The size of fully grown erythrocytic meronts exceeds that of the nuclei of infected erythrocytes (Fig. 1c). Fully grown gametocytes are roundish, oval or of irregular form, and their size exceeds that of the nuclei of infected erythrocytes (Fig. 4t-x). Pedunculated oocysts are absent

c Exo-erythrocytic merogony takes place in cells of the reticuloendothelial system. Erythrocytic trophozoites and growing meronts contain scanty cytoplasm (Fig. $3 \mathrm{~g}$ ). Growing erythrocytic meronts are nucleophilic. The size of fully grown erythrocytic meronts does not exceed that of the nuclei of infected erythrocytes (Fig. $3 \mathrm{~g}$, s). Fully grown gametocytes are roundish, oval, of irregular form, sometimes oval-elongated; their size does not exceed that of the nuclei of infected erythrocytes (Fig. $5 \mathrm{~m}$ ). Pedunculated oocysts are present. Subgenus Bennettinia contains only one species, Plasmodium juxtanucleare [81]

d Exo-erythrocytic merogony takes place in cells of the reticuloendothelial system. Erythrocytic trophozoites (Fig. 3d) and growing meronts (Fig. 3x) contain plentiful cytoplasm. The size of fully grown erythrocytic meronts exceeds that of the nuclei of infected erythrocytes (Figs. 2d, e; $3 w$, y). Fully grown gametocytes are elongated (Figs. 4c-s; 5i, k, o). Pedunculated oocysts are absent

e Exo-erythrocytic merogony takes place in cells of the reticuloendothelial system. Erythrocytic trophozoites (Fig. 3a, b) and growing meronts (Fig. 3e-j) contain scanty cytoplasm. The size of fully grown erythrocytic meronts does not exceed or only slightly exceeds that of the nuclei of infected erythrocytes occasionally (Fig. 3p-r). Fully grown gametocytes are elongated (Fig. 4c-r). Pedunculated oocysts are absent

birds gain Novyella infections in their wintering grounds and transport them to their breeding grounds where they are normally not transmitted [8, 71-73]. Factors preventing spread of Novyella infections globally are unclear. Novyella species are the most poorly studied group of avian malaria pathogens, with nearly no information available about exo-erythrocytic development, virulence, sporogony and vectors for the great majority $[1,4,8$, 72]. A few Novyella parasites (P. vaughani, Plasmodium rouxi, Plasmodium homopolare) are actively transmitted in countries with temperate climates, but they are absent or of low prevalence in areas with cold climates located close to the Polar Circles $[1,8,18,19,58,68]$.

Limited available experimental information indicates that some Novyella species ( $P$. ashfordi, $P$. rouxi) may cause severe and even lethal malaria in some birds due to blood pathology $[1,8,74,75]$, but the complete mechanism of their pathogenicity remains unresolved, mainly due to lack of information about exo-erythrocytic development [72]. Investigation of life cycles and virulence of infections caused by Novyella species is an important task in current avian malaria research. 
Many species of Plasmodium inhabit numerous species of birds and use mosquitoes of different genera for transmission $[1,8,9,11]$. Within this spectrum of hosts and vectors, the same parasite species might exhibit diverse morphological forms and strain varieties. Because of these morphological variants, it has been conventional in old avian malaria research (approximately between 1927 and 1995) that any new Plasmodium species description should only be accepted if supported by a comprehensive package of taxonomic features, which not only included the full range of blood stages, but also data on the vertebrate host specificity, periodicity of erythrocytic merogony, tissue merogony, vectors and patterns of sporogonic development. It is not surprising that recent molecular studies supported the validity of the old Plasmodium species descriptions, which were detailed and precise (Table 7). Application of molecular diagnostic tools in studies of avian haemosporidian parasites [29, 69, 76, 77] opened new opportunities to distinguish haemosporidian parasites based on their unique DNA sequences. This stimulated biodiversity research of wildlife Plasmodium parasites, particularly because the molecular characterization, which was done in parallel with morphological description of blood stages, made each parasite species detection readily repeatable at all stages of life cycle (Table 7).

A list of synonymous names of avian Plasmodium species and the justification of the nomenclature status of these names are given in Table 8. The majority of these parasite descriptions are insufficiently complete and were not accompanied with molecular characterization. Due to the huge genetic diversity of avian malaria pathogens and numerous genetic lineages reported in birds, some of these names might be validated in the future, and they represent a reserve for future taxonomic work. However, available descriptions of these parasites do not provide sufficient information to readily distinguish them from parasites, whose validity is well established (Tables $3,4,5$, 6). For clearness of scientific texts, it is preferable to avoid use of the synonymous names before additional data on their validity are available. Reports of parasite lineages and GenBank accessions of their DNA sequences in publications would be helpful to specify Plasmodium species identity in the future.
A list of the Plasmodium species names of unknown taxonomic position (incertae sedis) and also the names of species of doubtful identity, which require further investigation (species inquirenda), is given in Table 9. All these parasite descriptions are insufficiently complete and were not accompanied with molecular characterization. Taxonomic status of the majority of these names was justified in [8]. Twenty names of Plasmodium parasites were added to this list and their taxonomic status was explained (Table 9). The majority of these parasite descriptions are based on preparations with co-infections of several Plasmodium parasites belonging to same and (or) different genera. This raises a question if all blood stages reported in the original descriptions truly belong to corresponding species.

Additionally, in many of such parasite descriptions, gametocytes were not described, but this stage is essential for the identification of some Plasmodium species (Tables 3, 4, 5, 6, Figs. 4, 5). It is important to note that the descriptions of many Plasmodium parasites, which were incorporated in Table 9 and published during past 15 years, contain some information about their blood stages. Additionally, the type material was designated in many descriptions, but usually is insufficient for practical use and distinguishing parasites at the species level, particularly because (1) the type preparations contain coinfections and (2) single cells (meronts) were designated as holotypes. Single cells usually do not reflect entire morphological diversity of malaria parasites, so deposition of parahapantotype material is preferable in wildlife haemosporidian research $[35,49,58,78]$. Validation of some names listed in Table 9 is possible in the future, but it requires additional research, preferably based on new samples from the same avian hosts and type localities.

Invalid Plasmodium parasite names (nomen nudum) are listed in Table 9. These names were not accompanied with descriptions so have no status in nomenclature. The names of this category can be used as a reserve for new parasite descriptions in the future, but it is preferable not to use them to avoid taxonomic confusion [78].

The subgenus Papernaia was created for Novyella-like avian malaria parasites, whose erythrocytic meronts do not possess globules (Fig. 3f, h-l), structures of unclear origin and function $[79,80]$. The feature of the presence or absence of such globules is used in distinguishing 


\section{Table 3 Key to the Haemamoeba species}

\begin{tabular}{|c|c|}
\hline Step & Features and species \\
\hline $1(16)$ & $\begin{array}{l}\text { Roundish or oval pigment granules predominate in gametocytes (Fig. } 4 \mathrm{u}-\mathrm{x} \text { ). Elongate rod-like in form pigment granules (Fig. } 5 \mathrm{n} \text { ) are absent, but single slightly } \\
\text { elongate pigment granules might occur occasionally }\end{array}$ \\
\hline $2(22)$ & A residual body (Fig. $5 \mathrm{~s}$ ) is absent in mature erythrocytic meronts. Of oval-elongate form gametocytes, which are over $10 \mu \mathrm{m}$ in length (Fig. $5 \mathrm{i}$ ), are present \\
\hline $3(17)$ & $\begin{array}{l}\text { Large ( } \geq 1 \mu \mathrm{m} \text { in diameter) vacuoles (Figs. } 2 \mathrm{~g}, \mathrm{~h}, 5 \mathrm{u} \text { ) are absent from growing erythrocytic meronts. Markedly vacuolated erythrocytic meronts (Fig. } 2 \mathrm{f}-\mathrm{h} \text { ) are } \\
\text { absent }\end{array}$ \\
\hline $4(9)$ & Maximum number of merozoites in mature (Figs. $2 e, j, 3 n-r, y, 5 r, s$ ) erythrocytic meronts is $\leq 12$ \\
\hline $5(8)$ & $\begin{array}{l}\text { Maturing and mature erythrocytic meronts enlarge infected erythrocytes }<1 / 2 \text { in area in comparison to uninfected erythrocytes (compare infected and unin- } \\
\text { fected erythrocytes in Fig. 2g, h, see also Fig. 5s); numerous mature meronts adhere to erythrocyte nuclei (Fig. 2j) }\end{array}$ \\
\hline $6(7)$ & $\begin{array}{l}\text { Merozoites locate haphazardly in mature meronts (Fig. 1c). Residuum cytoplasm (Fig. 5s) is invisible in mature meronts, and merozoites never appear to have } \\
\text { connections to the residuum cytoplasm }\end{array}$ \\
\hline
\end{tabular}

7 (6) Nuclei locate on periphery of maturing and mature meronts (Fig. 5r). Residuum cytoplasm is visible and locates centrally in maturing meronts (Fig. 5 r). Maturing merozoites have connections to the residuum cytoplasm, and these connections look like small wisps of cytoplasm extending towards merozoites (Fig. $5 \mathrm{r}$ )

$$
\text { ... parvulum [51] }
$$

8 (5) Maturing and mature meronts enlarge infected erythrocytes over $1 \frac{1}{2}$ in area in comparison to uninfected erythrocytes (Fig. $5 \mathrm{p}$ ). The majority of maturing meronts are rounded in shape, they locate away from erythrocyte nuclei, which are markedly displaced toward erythrocyte envelope from earliest stages of meronts development (Fig. 5p)

$$
\text { P. caloti }{ }^{\mathrm{a}}[57]
$$

Maximum number of merozoites in mature erythrocytic meronts is $>12$. Mature meronts and gametocytes are large (size is significantly greater than erythrocyte nuclei); they occupy $>1 / 2$ of the cytoplasm in infected erythrocytes (Figs. 2j; $4 u-x$ )

10 (23) Pigment granules in gametocytes do not tend to be clumped in a spot, which is usually located near a margin of the parasite (Fig. 4t, w). If present occasionally, such position of pigment granules does not predominate in mature gametocytes

11 (10) Pigment granules in mature gametocytes show markedly different patterns of position in the cytoplasm; they often are randomly scattered (Figs. 1b, 5v), but also might be variously grouped (Fig. 5t) and even aggregated in solid masses (Fig. 4u, v, x)

12 (15) Largest fully grown gametocytes can occupy all available cytoplasmic space in infected erythrocytes (Fig. 5x, y). Length of the largest gametocytes exceed $10 \mu \mathrm{m}$

13 (14) Development in the blood is asynchronous, with all blood stages present in circulation simultaneously. Periodicity of erythrocytic merogony is 36 h; Specific parasite of domestic chicken. Passeriform birds are resistant. In the nature, transmission does not occur outside the Oriental zoogeographical region

$$
\text { ... gallinaceum }[1,8,83]
$$

14 (15) Development in the blood is synchronous, with not all blood stages present in circulation simultaneously. Periodicity of erythrocytic merogony is $24 \mathrm{~h}$. Domestic chicken was reported to be resistant. In the nature, transmission occurs outside the Oriental zoogeographical region

$$
\text { .. P. coturnixi }[8,84]
$$

15 (12) Largest fully-grown gametocytes do not occupy all available cytoplasmic space in infected erythrocytes; a small non-occupied space is usually visible in infected erythrocytes (Fig. $4 u-x$ ). Length of the largest gametocytes does not exceed $10 \mu \mathrm{m}$. Domestic chicken is resistant. Development in the blood is asynchronous, with all blood stages (trophozoites, growing and mature meronts as well gametocytes) present in blood films simultaneously. Periodicity of erythrocytic merogony is $36 \mathrm{~h}$

\section{P. relictum $[8,26,85]$}

16 (1) Pigment granules in gametocytes are roundish, oval and elongate rod-like (Fig. 5n). Rod-like pigment granules are common and might predominate in microgametocytes (Fig. $5 \mathrm{n}$ ), but they are less common and often do not predominate in macrogametocytes

$$
\text { P. cathemerium }[1,8,86]
$$

17 (3) Large ( $\geq 1 \mu \mathrm{m}$ in diameter) vacuoles (Figs. $2 \mathrm{~h} ; 5 \mathrm{u}$ ) are common in erythrocytic meronts

18 (21) One or several large vacuoles, which do not exceed $2 \mu \mathrm{m}$ in diameter, are often present in growing erythrocytic meronts. Markedly vacuolated erythrocytic meronts are common (Fig. 2f-h). Pigment granules do not gather around these vacuoles. Trophozoites lack large (>1 $\mu \mathrm{m}$ in diameter) vacuoles. Lobulated in form gametocytes (Fig. 4x) are absent or develop only occasionally

19 (20) Vacuoles are absent or occur occasionally in erythrocytic trophozoites. Pigment granules in fully grown gametocytes distinctly vary in size, and small (<0.5 $\mu \mathrm{m})$ and medium $(0.5-1.0 \mu \mathrm{m})$ size granules occur simultaneously (Fig. $5 \mathrm{v}$ ). The medium-size pigment granules are common (Fig. 5v). Phanerozoites do not develop in brain of domestic canaries

P. giovannolai $[1,8,87]$

20 (19) Vacuoles often present in erythrocytic trophozoites. Pigment granules in fully grown gametocytes are more or less similar in size, usually they are small $(<0.5 \mu \mathrm{m})$ (Fig. 5t). Medium-size $(0.5-1.0 \mu \mathrm{m})$ pigment granules (Fig. 5v) might occur, but are not characteristic. Phanerozoites develop in brain of domestic canaries

.. P. matutinum $[1,8,66,88]$

21 (19) Each advanced trophozoites possess one large ( $>1 \mu \mathrm{m}$ in diameter) roundish centrally located vacuole. One large ( $>2 \mu \mathrm{m}$ in diameter) vacuole is present in growing erythrocytic meronts (Fig. 5u). Pigment granules gather around this vacuole. Lobulated in form gametocytes (Fig. 4x) are common 
Table 3 (continued)

\begin{tabular}{|c|c|}
\hline Step & Features and species \\
\hline \multirow[t]{2}{*}{$22(2)$} & $\begin{array}{l}\text { A residual body (Fig. } 5 \text { s) is present in mature erythrocytic meronts. Of oval-elongate form gametocytes, which are over } 10 \mu \mathrm{m} \text { in length (Fig. } 5 \mathrm{i} \text { ), are present. } \\
\text { Growing erythrocytic meronts often possess vacuoles (Fig. } 2 \mathrm{~g}, \mathrm{~h} \text { ) }\end{array}$ \\
\hline & ...P. griffithsi $[1,8]$ \\
\hline $23(10)$ & $\begin{array}{l}\text { Pigment granules in gametocytes clearly tend to be clumped in a spot, which is located near a margin of the parasite (Fig. } 4 \mathrm{t} \text {, w). Such position of pigment } \\
\text { granules predominates in mature gametocytes. Pigment granules can be aggregated into a solid mass of pigment, which also usually locates near a margin of } \\
\text { the parasite }\end{array}$ \\
\hline & P. lutzi $[8,90,91]$ \\
\hline
\end{tabular}

a Plasmodium caloti was described from the Eurasian skylarks Alauda arvensis co-infected with several other Plasmodium species, and this races a question if all blood stages (particularly gametocytes), which were reported in the original description [57], truly belong to this parasite. However, because of (1) the marked influence on host cell (marked enlargement of infected erythrocytes and displacement of their nuclei) and (2) the relatively regular rounded form and smooth margins of mature meronts (Fig. 5p), which produce small number of merozoites $<10$ ), this parasite is morphologically unique and can be distinguished from other Haemamoeba species. The original description is fragmentary [57], and re-description of this parasite is needed

\section{Table 4 Key to Giovannolaia species}

\section{Step Features and species}

1 (16) Elongate meronts, which grow laterally to nuclei of infected erythrocytes (Figs. 2c-e; 3w-y), predominate

2 (3) Cytoplasm of gametocytes (especially macrogametocytes) is highly vacuolated (Fig. 4o). Large (>1.5 $\mu$ m in diameter) vacuoles are present in some macrogametocytes

P. fallax $[1,8,92]$

3 (2) Cytoplasm of gametocytes is not highly vacuolated; if vacuoles are present in macrogametocytes, they are few and of small size (< $1 \mu m$ in diameter) (Fig. $4 \mathrm{p}-\mathrm{s})$

4 (5) Pigment granules in the majority of erythrocytic meronts are aggregated into large (> $1.5 \mu \mathrm{m}$ in length) clumps, which usually locate at one end of elongate meronts (Fig. 5f)

P. anasum $[1,8,93]$

5 (4) Pigment granules in the majority of erythrocytic meronts are not aggregated into large (> $1.5 \mu \mathrm{m}$ in length) clumps, which usually locate at one end of elongate meronts (Fig. 5f). Location of pigment granules in erythrocytic meronts is markedly variable

6 (7) Nuclei tend to lean to one end in the majority of growing erythrocytic meronts (Fig. 5d)

$$
\text { P. leanucleus }[8,94]
$$

7 (6) Nuclei do not tend to lean to one end in the majority of growing erythrocytic meronts (Fig. 5d). Position of nuclei in developing meronts is markedly variable (Fig. $3 u, x)$

8 (11) Average number of merozoites in mature meronts is $<12$

9 (10) Fully grown erythrocytic meronts and gametocytes are thin slender cells, they do not displace the nuclei of infected erythrocytes and usually do not adhere to the nuclei (Fig. 5e)

$$
\text { P. gundersi }[1,8,95]
$$

10 (9) Fully grown erythrocytic meronts (Fig. 3w) and gametocytes (Fig. 5k) are broad cells, which width is equal to the width of erythrocyte nuclei or is greater; both mature meronts and gametocytes displace the nuclei of infected erythrocytes laterally and often adhere to the nuclei (Figs. 3w; 5k)

$$
\text { P. octamerium }[8,96]
$$

11 (8) Average number of merozoites in mature meronts is $\geq 12$

12 (15) Gametocytes and meronts grow around nuclei of erythrocytes (Figs. 2c, d; 4r, s). Fully grown erythrocytic meronts and gametocytes usually only slightly (if at all) influence infected erythrocytes and do not displace or only slightly displace nuclei of erythrocytes laterally (Figs. $2 \mathrm{~d}$, e: 4s). Infected erythrocytes usually do not become rounded (Fig. 5x)

13 (14) Fully-grown erythrocytic meronts (Fig. 2d, e) and gametocytes (Fig. 4s) markedly (often nearly completely or completely) encircle nuclei of infected erythrocytes; completely circumnuclear mature meronts and gametocytes frequently develop, but their occurrence depends of stage of parasitemia, so they might be not always seen in blood films

\section{P. circumflexum $[1,8,97]$, P. homocircumflexum $[35]^{\text {a }}$}

14 (13) Fully grown erythrocytic meronts never assume circumnuclear form (Fig. 3w, y). Gametocytes nearly completely (Fig. 4r) or completely (Fig. 4s) encircle nuclei of infected erythrocytes; completely circumnuclear mature gametocytes develop, but usually are rare. Advanced trophozoites and young meronts often possess large (> $1 \mu \mathrm{m}$ in diameter) vacuoles (Fig. 5j) 
Table 4 (continued)

\section{Step Features and species}

15 (12) Gametocytes and meronts start to grow around nuclei of erythrocytes

However, fully grown meronts markedly displace nuclei of erythrocytes and assume various irregular forms; they often roundish or close to roundish in shape (Fig. $5 \mathrm{w}$ ), markedly displace the nuclei of infected erythrocytes and can occupy all available cytoplasmic space in the erythrocytes (Fig. 5w). Fully grown gametocytes markedly deform infected erythrocytes, which become rounded (Fig. 5x, y)

\section{P. gabaldoni $[8,99]$}

16 (1) Elongate erythrocytic meronts, which grow laterally to nuclei of infected erythrocytes (Figs. 2c-e; 3w-y), are absent or appear only occasionally; they never predominate. The majority of fully grown meronts are of roundish, oval or irregular form; they do not take or take only occasionally the lateral position to nuclei of erythrocytes (Fig. $5 q-s$ )

17 (24) Large (> $1.5 \mu \mathrm{m}$ in diameter) vacuoles (Fig. $5 \mathrm{~g}$ ) absent from gametocytes. If small vacuoles are present in gametocytes, pigment granules do not gather around vacuoles

18 (25) Fully grown gametocytes do not tend to lie obliquely in infected erythrocytes (Fig. 5i, o), and they do not displace the nuclei towards one pole of the erythrocytes

19 (26) Growing erythrocytic meronts do not produce long (>2 $\mu \mathrm{m}$ in length) tail-like or finger-like outgrowths (Fig. 5c)

20 (21) Erythrocytic meronts take a polar or subpolar position in infected erythrocytes, and their influence on infected erythrocytes is usually not pronounced (Fig. 3r)

\section{P. polare $[1,8,100]$}

21 (20) Erythrocytic meronts can be seen anywhere in infected erythrocytes including a lateral, subpolar and polar position. If meronts take a polar or subpolar position in the erythrocytes, they markedly influence the host cells causing their deformation and (or) displacement of their nuclei

22 (23) Maximum number of merozoites in mature meronts $>10$. Size of pigment granules in macro- and microgametocytes is clearly different P. pinottii $[1,8,101]$

23 (22) Maximum number of merozoites in mature meronts $<10$. Size of pigment granules in macro- and microgametocytes is similar P.garnhami $[1,8,102]$

24 (17) Large (> $1.5 \mu \mathrm{m}$ in diameter) vacuoles (Fig. 5g) develop in many macrogametocytes. Pigment granules often gather around these vacuoles P. formosanum $[1,8,103]$

25 (18) Fully grown gametocytes tend to lie obliquely in infected erythrocytes, and they displace the nuclei towards one pole of the erythrocytes (Fig. 50)

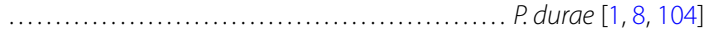

26 (19) Growing erythrocytic meronts often produce long (>2 $\mu \mathrm{m}$ in length) tail-like or finger-like outgrowths (Fig. 5c)

27 (28) Nuclei in mature erythrocytic meronts are usually arranged as fans (Fig. 3v), rosettes (Fig. 5r), or more or less pronounced rows (Fig. 3w). Infected erythrocytes with segmented mature meronts are often rounded (Fig. 5r). Fully grown gametocytes do not fill erythrocytes up to their poles (Fig. 4h)

P. pedioecetae $[1,8,105,106]$

28 (27) Nuclei in mature erythrocytic meronts are usually located randomly (Fig. 3q) and they only occasionally can be arranged as rosettes. Infected erythrocytes with segmented mature meronts are not rounded (Fig. 3w). Fully grown gametocytes fill erythrocytes up to their poles (Fig. 5k)

P. hegneri $[8,93]$

a Based on available information, $P$. circumflexum and $P$. homocircumflexum are cryptic species, which cannot be distinguished using morphological features of their blood stages [35]. Cytochrome $b$ sequences can be used to distinguish these infections (see Table 7) 


\section{Table 5 Key to the Novyella species}

\section{Step Features and species}

1 (19) Maximum number of merozoites in erythrocytic meronts $>4$

2 (41) Maturing erythrocytic meronts, which displace host-cell nuclei, assume a fan-like shape and possess elongate nuclei (Fig. 3v), are absent

3 (26) Erythrocytic meronts, which lie free in the cytoplasm of host cell and do not touch the nuclei of infected erythrocytes (Fig. 3e-l, o), are present

4 (32) Trophozoites and binuclear meronts (Fig. 3e, f) do not produce clearly defined long outgrowths (Fig. 5a); if ameboid outgrowths are present, they do not exceed the main body of the trophozoites in length

5 (42) Ends of growing macrogametocytes are similar in width (Fig. 4c-e, g-r)

6 (9) Number of merozoites in mature erythrocytic meronts is relatively stable. Over $90 \%$ of the mature meronts contain 6 merozoites

7 (8) Macrogametocyte nuclei are terminal in position (Fig. 4g). Refractive globules (Fig. 3h) are present in erythrocytic meronts

$$
\text { P. parahexamerium [55] }
$$

8 (7) Macrogametocyte nuclei are central or subcentral in position (Fig. 40). Refractive globules are absent from erythrocytic meronts (Fig. 3p)

$$
\text { ... P. hexamerium }[1,8,55,107]
$$

9 (6) Number of merozoites in mature erythrocytic meronts is variable

10 (37) Growing and mature meronts assume various positions to the erythrocyte nuclei; they can be found in polar, sub-polar and lateral position in relation to the host cell nuclei

11 (38) Binuclear erythrocytic meronts do not possess large (of size, which is similar to nuclei of the meronts), centrally located vacuoles (Fig. 3e). Macro- and microgametocytes are of similar shape, they assume similar positions in erythrocytes (Fig. 4g, h)

12 (39) Gametocytes do not possess refractive globules

13 (16) Erythrocytic meronts possess globules in natural infections (Fig. 3f, h-j)

14 (15) The majority of trophozoites as well as developing and mature erythrocytic meronts possess one of circular shape, prominent (on average $0.5 \mu \mathrm{m}^{2}$ in area) pigment granule (Fig. 3q). Fan-like in shape mature meronts predominate

$$
\text { .. P. unalis [49] }
$$

15 (14) The majority of trophozoites, developing and mature erythrocytic meronts possess 1-4 (usually $2-3$ ) small $\left(<0.5 \mu m^{2}\right.$ in area), of different size pigment granules (Fig. 3c, h). Fan-like in shape mature meronts (Fig. 3o) are uncommon

$$
\text { P. vaughani }[1,8,49,55,108]
$$

16 (13) Erythrocytic meronts do not possess globules in natural infections (Fig. 3g, n, o, r, s)

17 (18) Fan-like mature meronts containing 7-8 merozoites are common (Fig. 30); pigment granules in gametocytes are clumped together into a prominent group, which is predominantly of terminal position in the gametocytes (Fig. 4e, h)

$$
\text { .. P. ashfordi [71] }
$$

18 (17) Fan-like mature meronts containing 7-8 merozoites are absent; pigment granules in gametocytes are scattered or clumped, but position of these clumps is irregular (never predominantly terminal) in the gametocytes

$$
\text { . P. forresteri }[8,109]
$$

19 (1) Maximum number of merozoites in erythrocytic meronts is 4

20 (21) Erythrocytic meronts do not possess globules in natural infections (Fig. 3g)

$$
\text { P. bertii }[8,110]
$$

21 (20) Erythrocytic meronts possess globules in natural infections (Fig. 3f, h-l)

22 (23) One small (<0.5 $\mu \mathrm{m}$ in diameter) refractive globule present in the majority of meronts (Fig. 3f, h, j). Blue non-refractive globules (Fig. $3 k$, I) are absent from meronts. The cytoplasm in gametocytes is more or less homogenous, but never is granular-like (Fig. 4c) or globular-like (Fig. 4l, m) in appearance

$$
\text { P. rouxi }[1,8,52,111]
$$

23 (22) Refractive globules (Fig. 3f, h-j) are absent from meronts. One blue non-refractive globule present in each advanced trophozoite (Fig. 3b), growing and mature meront (Fig. 3k, I). The cytoplasm in gametocytes is granular-like (Fig. 4c) or globular-like in appearance (Fig. 4l, m)

24 (25) One large (size similar to parasite nuclei or greater) blue non-refractive globule present in each advanced trophozoite (Fig. 3b), growing and mature meront (Fig. 3l). The cytoplasm in macro- and microgametocytes is markedly globular-like in appearance (Fig. 4l, m). Average number of pigment granules in macroand microgametocytes is close to 10

$$
\text { P. megaglobularis [52] }
$$

25 (24) One small (size smaller than parasite nuclei) blue non-refractive globule present in each advanced trophozoite, growing and mature meront (Fig. 3k). The cytoplasm in gametocytes is markedly granular in appearance, which is better visible in macrogametocytes (Fig. 4c); globular-like appearance of the cytoplasm (Fig. $4 \mathrm{l}, \mathrm{m}$ ) is not characteristic. Average number of pigment granules in macro- and microgametocytes is close to 5

$$
\text { P. globularis [52] }
$$

26 (3) Erythrocytic meronts, which lie free in the cytoplasm of host cell and do not touch the nuclei of infected erythrocytes (Fig. 3e-l, o), are absent. Erythrocytic meronts are strictly nucleophilic (Figs. $3 n, 5 ; 5 q)$

27 (40) Both meronts (Fig. 3n, p, s, t) and gametocytes (Fig. 4a, d) are strictly nucleophilic

28 (29) Large ( $\geq 1 \mathrm{~m} \mu$ in length) pigment granules are present in gametocytes (Fig. 4d); both mature gametocytes (Fig. 4d) and meronts (Fig. 3n, t) do not displace nuclei of erythrocytes 
Table 5 (continued)

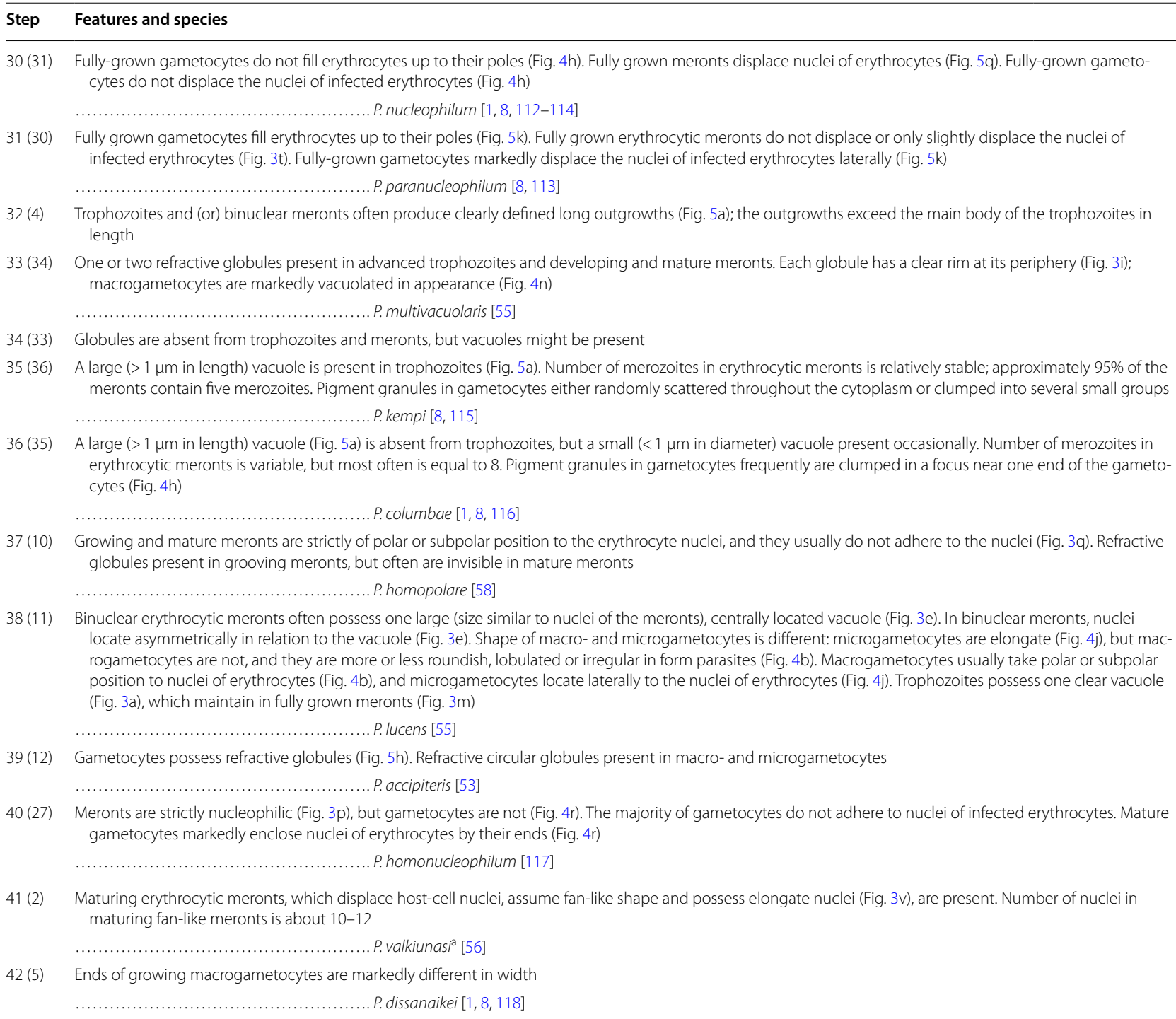

a Plasmodium valkiunasi was described from Eurasian magpies Pica pica co-infected with several other Plasmodium species, and this races a question if all blood stages (particularly gametocytes), which were reported in the original description [56], truly belong to this species. However, this parasite is morphologically unique and can be distinguished from other Novyella species because of unique shape of its maturing meronts (Fig. 3v), which are large, develop in mature erythrocytes, have a regular fan-like form and possess numerous (about 12) peripherally located elongate nuclei. The original description is fragmentary [56], and re-description of this parasite is needed

some species of malaria parasites belonging to subgenus Novyella during natural infections (Table 5). It is interesting to note that experimental studies with a Plasmodium ashfordi (pGRW2) strain, which normally do not possess globules in erythrocytic meronts, show that the globules appeared in this parasite's meronts after several artificial passages in unusual avian hosts. This strain was originally isolated from the Common cuckoo Cuculus canorus (Cuculiformes), and it did not possessed globules in erythrocytic meronts [75] in the cuckoo or during the first passage in the Eurasian siskin Carduelis spinus. However, the globules appeared in the meronts of the 
Table 6 Key to Huffia species

\section{Step Features and species}

1 (2) Development and maturation of gametocytes occurs in various immature red blood cells, including erythroblasts (Fig. 4y). The outline of nuclei in growing erythrocytic meronts (Fig. $2 \mathrm{~m}, \mathrm{n}$ ) as well as growing and mature gametocytes (Fig. 4y) is smooth, and boundaries between nuclei and the cytoplasm are strictly distinct (Figs. 2m, n; 4y)

$$
\text { P. polymorphum [59] }
$$

2 (1) Development and maturation of gametocytes occurs only in mature or nearly mature red blood cells (Fig. 4p, q); gametocytes do not develop in erythroblasts. The outline of nuclei in growing erythrocytic meronts (Fig. 2l, o, p) as well as growing and mature gametocytes (Fig. 4p, q) is markedly variable, predominantly not smooth, and boundaries between the nuclei and the cytoplasm are often poorly distinct (Figs. 20, p: $4 p, q)$, particularly in the growing parasites

3 (6) In peripheral blood, trophozoites and erythrocytic meronts develop mainly in young red blood cells. Maximum number of merozoites in erythrocytic meronts is less than 20

4 (5) Elongated erythrocytic merozoites are present (Fig. 2k). Fully grown gametocytes are slender; they do not displace or only slightly displace the nuclei of infected erythrocytes laterally (Fig. 4p, q). Maximum width of fully grown gametocytes is equal or less than the width of nuclei of host cells (Fig. 4p, q)

P. elongatum $[1,8,23,119,120]$

5 (4) Elongated erythrocytic merozoites (Fig. 2k) are absent. Fully grown gametocytes are broad; they markedly displace the nuclei of infected erythrocytes laterally and can fill the poles of infected erythrocytes completely (Fig. 5l). Maximum width of fully grown gametocytes is greater than the width of nuclei of host cells (Fig. 5l)

$$
\text { P. hermani }[8,120,121]
$$

6 (3) In peripheral blood, trophozoites and erythrocytic meronts develop mainly in mature red blood cells. Maximum number of merozoites in erythrocytic meronts is greater than 20

$$
\text { P. huffi }[1,8,122]
$$

\begin{tabular}{|c|c|c|}
\hline Parasite subgenus and species & GenBank accession and lineage code (in parentheses) $^{a}$ & References $^{b}$ \\
\hline \multicolumn{3}{|l|}{ Haemamoeba } \\
\hline P. caloti & Not available & Not available \\
\hline P. cathemerium & AY377128 (pSEIAUR01) & [123] \\
\hline P. coturnixi & Not available & Not available \\
\hline P.gallinaceum & AY099029 (pGALLUS01) & {$[124]$} \\
\hline P. giovannolai & Not available & Not available \\
\hline P. griffithsi & Not available & Not available \\
\hline P. lutzi & KC138226 (pTFUS05) & [91] \\
\hline P. matutinum & KY287235 (pLINN1) & {$[66]$} \\
\hline P. parvulum & Not available & Not available \\
\hline P. subpraecox & Not available & Not available \\
\hline P. relictum & $\begin{array}{l}\text { AF495571 (pSGS1), AY831748 (pGRW11), AY099041 (pGRW4), KC342644 (pLZ- } \\
\text { FUS01), MG724747 (pPHCOL01) }\end{array}$ & {$[67,71,117]$} \\
\hline P. tejerai & JX272844 (pSPMAG01) & {$[50]$} \\
\hline \multicolumn{3}{|l|}{ Giovannolaia } \\
\hline P. anasum & Not available & Not available \\
\hline P. circumflexum & AF495576 (pTURDUS1) & {$[67]$} \\
\hline P. durae & Not available & Not available \\
\hline P. fallax & Not available & Not available \\
\hline P. formosanum & Not available & Not available \\
\hline P.gabaldoni & Not available & Not available \\
\hline P.garnhami & Not available & Not available \\
\hline P. gundersi & Not available & Not available \\
\hline P.hegneri & Not available & Not available \\
\hline P. homocircumflexum & KC884250 (pCOLL4) & [35] \\
\hline
\end{tabular}

Table 7 Mitochondrial cytochrome $b$ sequences, which have been developed for molecular detection and identification (barcoding) of avian Plasmodium parasites 
Table 7 (continued)

\begin{tabular}{|c|c|c|}
\hline Parasite subgenus and species & GenBank accession and lineage code (in parentheses) $^{a}$ & References $^{\mathbf{b}}$ \\
\hline P. leanucleus & Not available & Not available \\
\hline P. lophurae & Not available & Not available \\
\hline P.polare & Not available & Not available \\
\hline P. octamerium & Not available & Not available \\
\hline P. pedioecetae & Not available & Not available \\
\hline P. pinottii & Not available & Not available \\
\hline \multicolumn{3}{|l|}{ Novyella } \\
\hline P. accipiteris & Not available & Not available \\
\hline P. ashfordi & AF254962 (pGRW2) & [71] \\
\hline P. bertii & Not available & Not available \\
\hline P. columbae & Not available & Not available \\
\hline P. delichoni & KU529943 (pCOLL6) & [72] \\
\hline P. dissanaikei & Not available & Not available \\
\hline P. forresteri & Not available & Not available \\
\hline P. globularis & EU770151 (pANLA1) & {$[52]$} \\
\hline P. hexamerium & Not available & Not available \\
\hline P. homonucleophilum & KC342643 (pSW2) & [117] \\
\hline P. homopolare & KJ482708 (pSOSP CA 3P) & [58] \\
\hline P. kempi & Not available & Not available \\
\hline P.lucens & FJ389156 (pCYOL2) & {$[55]$} \\
\hline P. megaglobularis & EU770152 (pCYOL1) & {$[52]$} \\
\hline P. multivacuolaris & FJ389157 (pANLA2) & {$[55]$} \\
\hline P. nucleophilum & JX467689 (pEG01) & [114] \\
\hline P. parahexamerium & FJ389155 (pALDI1) & {$[55]$} \\
\hline P.paranucleophilum & Not available & Not available \\
\hline P. rouxi & HM146901 (pPADOM16) & [68] \\
\hline P. unalis & KC771247 (pTFUS06) & [49] \\
\hline P. valkiunasi & Not available & Not available \\
\hline P. vaughani & DQ847271 (pSYAT05) & [22] \\
\hline \multicolumn{3}{|l|}{ Bennettinia } \\
\hline P. juxtanucleare & AB250415 (pGALLUS02) & {$[125]$} \\
\hline \multicolumn{3}{|l|}{ Huffia } \\
\hline P. elongatum & DQ368381 (pGRW6); KT282462 (pERIRUB01) & {$[23,120]$} \\
\hline P. hermani & Not available & Not available \\
\hline P. huffi & Not available & Not available \\
\hline P.polymorphum & Not available & Not available \\
\hline
\end{tabular}

a Only DNA sequences, for which parasite species identity was supported by morphological analysis are included in this table

b References of articles containing discussion of molecular characterization and morphological features of parasite species

same lineage after 3-4 passages via the infected blood inoculation in passeriform birds (G. Valkiūnas, unpublished). Molecular testing showed that the parasite lineage was the same. Pictures of erythrocytic meronts of the same isolate of the lineage pGRW2 in the Common cuckoo (Fig. 6a) and after the first passage in the Eurasian siskin Carduelis spinus (Passeriformes) (Fig. 6b) and several subsequent passages in siskins (Fig. 6c, d) illustrate this change. These experimental data indicate that malaria parasites which do not possess globules in 
Table 8 List of synonyms of Plasmodium species of birds

\begin{tabular}{|c|c|}
\hline $\begin{array}{l}\text { Synonymous name and references of original } \\
\text { description }\end{array}$ & Valid name $^{\mathrm{a}}$ \\
\hline Plasmodium alaudae [126] & P. relictum (partim) \\
\hline P. alloelongatum [53] ${ }^{\mathrm{b}}$ & P. elongatum \\
\hline P. bioccai $[56]^{c}$ & P. relictum \\
\hline P. biziurae [127] & P. relictum \\
\hline P. capistrani [128] & P. relictum \\
\hline P. centropi [129] & P. cathemerium (partim) \\
\hline P. chloropsidis [130] & P. relictum \\
\hline P. coluzzii $[57]^{c}$ & P. relictum \\
\hline P. dorsti $[56]^{c}$ & P. relictum \\
\hline P. ginsburgi $[57]^{c}$ & P. relictum \\
\hline P. heroni [131] & P. circumflexum \\
\hline P. huffi [122] & P. nucleophilum \\
\hline P.japonicum [132] & P. juxtanucleare \\
\hline P. metastaticum [133] & P.gallinaceum \\
\hline P. grassii [134] & P. relictum \\
\hline P. inconstans [86] & P. relictum \\
\hline P. maior [135] & P. relictum \\
\hline P. merulae [136] & P. vaughani \\
\hline P. mohammedi $[54]^{d}$ & P. rouxi \\
\hline P. muniae [137] & P. relictum \\
\hline P. majoris [138] & P. relictum (partim) \\
\hline P. oti [139] & P. hexamerium \\
\hline P. passeris [140] & P. relictum \\
\hline P.paddae [141] & P. relictum \\
\hline P. pericrocoti [142] & P. relictum \\
\hline P. ploceii [142] & P. relictum \\
\hline P. relictum quentini $[57]^{c}$ & P. relictum \\
\hline P. spheniscidae [143] & P. relictum \\
\hline P. tumbayaensis [144] & P. vaughani \\
\hline P. tenuis [145] & P. vaughani \\
\hline Plasmodium wasielewskii [146] & P. subpraecox \\
\hline
\end{tabular}

a Plasmodium species synonymous names published before 2000 were justified in [8]

b According to the original description [53], P. alloelongatum is similar to $P$. elongatum, but differs from the latter species mainly due to two characters: (1) the erythrocytic meront progeny is limited to 6 (predominantly 6-12 in $P$. elongatum), and (2) the undulating or rugged outlines and tapering gametocyte ends, which might extend into a distal spine or filaments. The irregularity of gametocyte shape (Fig. 4p) and presence of ameboid outgrowth (Fig. 4q) has been reported and illustrated in $P$. elongatum (the closely related lineages pGRW6 and pERIRUBO1), but have rarely pointed out in descriptions of this parasite $[1,23,120]$. Furthermore, the ameboid outgrowths in gametocytes were seen in the neohapantotype of P. elongatum (blood slide no. 216, the Natural History Museum, London). The number of nuclei in mature erythrocytic meronts is variable in P. elongatum during development in different host cells and avian hosts, and it is often $\leq 6[1,23,120]$. Plasmodium elongatum has been characterized molecularly (Table 7), and it has been reported in numerous bird species belonging to different orders both by microscopic examination of blood films and PCR-based testing, including species of Accipitriformes and Falconiformes [21]. Based on available information, Plasmodium alloelongatum cannot be distinguished is considered as a synonym of $P$. elongatum

c Observation of blood stages in various bird species experimentally infected with single infections of Plasmodium relictum lineages pSGS1 and pGRW11, which are closely related and widespread in Europe, show that main reported $P$. bioccai, P. coluzzii, P. dorsti, P. ginsburgi, P. relictum quentini blood stages (meronts and gametocytes) are present in these parasite lineages $[67,71,117]$. These

\section{Table 8 (continued)}

parasites were described in co-infection with Haemamoeba parasites, including P. relictum, and description of blood stages were fragmentary [56, 57]. Blood stages of all these parasites do not have unique characters, which could help to distinguish them from $P$. relictum. Plasmodium bioccai, $P$. coluzzii, $P$. dorsti, $P$. ginsburgi, $P$. relictum quentini are considered as synonyms of $P$. relictum

d Synonymous status of $P$. mohammed $i$ was specified in Table 9 (see the footnote "f")

Table 9 List of species names of bird malaria parasites belonging to the categories of nomen nudum, nomen dubium, species inquirenda and incertae sedis

\begin{tabular}{|c|c|}
\hline Name and references & Status $^{\mathrm{a}}$ \\
\hline Plasmodium alaudae $[57,126]^{\mathrm{b}}$ & Species inquirenda \\
\hline P. arachnidi [147] & Species inquirenda \\
\hline P. bambusicolai [148] & Species inquirenda \\
\hline P. beaucournui $[56]^{\text {b }}$ & Species inquirenda \\
\hline P. bigueti $[65]^{\mathrm{b}}$ & Species inquirenda \\
\hline P. buteonis $[53]^{c}$ & Species inquirenda \\
\hline P. coggeshalli $[149]^{b}$ & Species inquirenda \\
\hline P. conturnixae [150] & Nomen nudum \\
\hline P. corradettii [151] & Nomen dubium \\
\hline P. danilewskyi $[152,153]$ & Incertae sedis \\
\hline Plasmodium dherteae $[56]^{\mathrm{b}}$ & Species inquirenda \\
\hline P.gallinulae [130] & Incertae sedis \\
\hline P. gambeli [3] & Nomen nudum \\
\hline P. ghadiriani $[56]^{\mathrm{b}}$ & Species inquirenda \\
\hline P. golvani [56] ${ }^{\mathrm{b}}$ & Species inquirenda \\
\hline P. herodiadis [130] & Species inquirenda \\
\hline P. holti [154] & Nomen nudum \\
\hline P.jiangi [155] & Species inquirenda \\
\hline P. jeanriouxi $[57]^{\mathrm{b}}$ & Species inquirenda \\
\hline P. lagopi [156] & Species inquirenda \\
\hline P. lairdi [157] & Nomen nudum \\
\hline P. lenoblei $[56]^{\mathrm{b}}$ & Species inquirenda \\
\hline P. malariae raupachi [158] & Incertae sedis \\
\hline P. manwelli [159] & Nomen nudum \\
\hline P. ninoxi $[160]^{d}$ & Species inquirenda \\
\hline P. noctuae $[3,126]$ & Species inquirenda \\
\hline P. pachysomum $[54]^{\mathrm{e}}$ & Species inquirenda \\
\hline P. papernai $[149]^{b}$ & Species inquirenda \\
\hline Plasmodium pfefferi [54] $]^{\mathrm{e}}$ & Species inquirenda \\
\hline P. praecox [152] & Nomen nudum \\
\hline P. reniai $[57]^{\mathrm{b}}$ & Species inquirenda \\
\hline P. rousseloti [161] & Species inquirenda \\
\hline P. rouxi, as published in $[54]^{f}$ & $\begin{array}{l}\text { Species inquirenda (probably a new Plasmo- } \\
\text { dium species) }\end{array}$ \\
\hline P. sergentorum $[54]^{e}$ & Species inquirenda \\
\hline P. snounoui $[56]^{b}$ & Species inquirenda \\
\hline P. spartani [162] & Nomen nudum \\
\hline P. stellatum [54] $]^{\mathrm{e}}$ & Species inquirenda \\
\hline P. struthionis [163] & Incertae sedis \\
\hline P. tranieri $[56]^{b}$ & Species inquirenda \\
\hline P. venkataramiahii [164] & Nomen nudum \\
\hline
\end{tabular}

a Nomenclature status of the species names published before 2000 was justified in [8] 


\section{Table 9 (continued)}

b Plasmodium beaucournui, P. bigueti, P. coggeshalli, P. dherteae, P. ghadiriani, P. golvani, P. jeanriouxi, P. lenoblei, P. papernai, P. reniai, P. snounoui, P. tranieri were named and described, and $P$. alaudae was re-described from individual birds co-infected with parasites belonging to subgenera Haemamoeba, Giovannolaia and Novyella [56, $57,65,149]$. The authors of the original descriptions have grouped the blood stages visible in blood films and attributed them to different species provisionally, which is particularly obvious in case of parasites with elongate gametocytes. This makes species description and validation of parasite names questionable. Only single cells (erythrocytic meronts) were selected as holotypes in these parasite descriptions. However, due to morphological variation of blood stages of Plasmodium and presence of parasites at different stages of growth in each blood film, such methodology of designation of the type material can work only in case of exceptionally distinctive cell characters, which is not the case in all these parasite descriptions, particularly belonging to subgenus Haemamoeba. Molecular characterization of all these parasites is unavailable. It is clear from the original descriptions, that many individual birds were infected by representatives of several subgenera. However, the reported blood stages were selected and attributed to certain species without providing convincing explanations, making identifications difficult or even impossible based on available information. Co-infections of Plasmodium parasites belonging to different subgenera are common in wildlife, and the described cases of co-infections with several malaria parasites are not unpredictable [45]. However, description of new species from such co-infections hardly possible if the unique morphological characters of blood stages are absent, which is the case with P. beaucournui, P. bigueti, P. coggeshalli, P. dherteae, $P$. ghadiriani, P. golvani, P. jeanriouxi, P. lenoblei, P. papernai, P. reniai, P. snounoui, P. tranieri and also in re-description of $P$. alaudae. These parasites are considered as species inquirenda. Recent molecular studies provided molecular markers for distinguishing blood stages of Plasmodium species (Table 7). Examination of blood films from experimental infections shows variations in morphological characters of same parasite lineages in different avian hosts, calling for careful application of minor differences in blood stage morphology in avian malaria parasite taxonomy, particularly during co-infections

c Based on available information [53], P. buteonis cannot be distinguished from $P$. circumflexum and other similar parasites of Giovannolaia (Plasmodium gabaldoni, Plasmodium homocircumflexum). The main feature, which has been noted to distinguish $P$. buteonis from $P$. circumflexum in the original description [53], is the presence up to 36 nuclei in mature erythrocytic meronts of the former. Plasmodium circumflexum produce less number of nuclei in mature meronts. However, the description of $P$. buteonis is based on high parasitemia (6.6\%), with numerous multiple infections of the same erythrocytes, so it is difficult to rule out that 2 mature meronts were present in same cell in case of so great number of merozoites. Additionally, parasite morphology often changes during high parasitemia, so such samples should be carefully used in taxonomical descriptions. Plasmodium buteonis might be a valid name, but more research is needed to prove its validity. Molecular characterization of this parasite is absent, but is essential to solve the question about its validity

d Plasmodium ninoxi was described from owl Ninox scutulata in co-infection with Haemoproteus sp. [160]. Only one erythrocyte with 2 binuclear growing meronts was detected; no other data about merogony in the blood were provided. Plasmodium ninoxi gametocytes were reported to be rounded. Based on available information, it seems that infected blood was exposed to air, which stimulated rounding-up of haemoproteid gametocytes [8], which were attributed to $P$. ninoxi. DNA sequence was provided (AY099035.1), and it belongs to Plasmodium sp. Plasmodium ninoxi description is incomplete. Re-description is needed, and it is possible due to available sequence information. The most similar cytb sequence belong to $P$. gallinaceum, $P$. relictum and $P$. circumflexum

e Descriptions of P. pachysomum, P. pfefferi, P. sergentorum, P. stellatum [54] are incomplete. Information about morphology of gametocytes is absent. Molecular characterization is unavailable. Species identification is questionable based on the available information

f Paperna et al. [54] published re-description of $P$. rouxi from non-type avian host (Alauda arvensis, Alaudidae instead of Passer hispaniolensis, Passeridae whose is the type host). The re-description is based on samples, which were collected beyond of the type locality (France, instead of Algeria which is the type locality). This contradicts the Article 75.3.6 of the International Code of Zoological Nomenclature [78]. Additionally, according to [54], the erythrocytic meronts of the parasite from $A$. arvensis do not possess refractive globules and gametocytes possess few tiny pigment granules (Figs. 8, 9 in [54]). These are not characters of $P$. rouxi, which was described by Sergent et al. [111]. Sergent's original material from Algiers labelled "2198, 26.4.28, Institut Pasteur d'Algérie" is available in the Natural History Museum, London. Examination of this blood film showed that numerous erythrocytic meronts of this parasite

\section{Table 9 (continued)}

possess refractive globules (Fig. 3f, j) and gametocytes possess few large (Fig. 4o) pigment granules. The latter character is an important feature of $P$. rouxi. Based on available information, the parasite described in [54] as 'P. rouxi' cannot be attributed to $P$. rouxi and is considered as a species inquirenda. The parasite described by Paperna et al. [54] is characterized by presence of (1) the relatively prominent cytoplasm in growing meronts and (2) tiny size of pigment granules in gametocytes, so might belong to a new Plasmodium species. Additional investigation is needed to answer this question. In the same study, Paperna et al. [54] described a new species Plasmodium mohammedi, which was reported, Passer domesticus (the common host of $P$. rouxi in Mediterranian region [68]). Blood stages of $P$. mohammedi are indistinguishable from $P$. rouxi [111], particularly due to the presence of refractive globules in erythrocytic meronts and large pigment granules in gametocytes (see Figs. 18-21 in [54]). Plasmodium mohammedi is a synonym of $P$. rouxi. Molecular identification of $P$. rouxi (lineage pPADOM16) was developed [68]. Application of the barcoding indicates that the details of disposition of nuclei in erythrocytic meronts during different infections, particularly in different avian hosts, is variable in P.rouxi, but binuclear "bow-tie" form parasites often are present (Fig. 3f) and can be used for this parasite species identification. Additionally, presence of few large pigment granules in mature gametocytes also is a characteristic feature, and it recommended to use for distinguishing P. rouxi infection (Fig. 4o) from other Novyella parasites producing tetranuclear erythrocytic meronts

natural hosts might develop this structure after artificial passages via infected blood inoculation in unusual avian hosts. In other words, this feature hardly can be used in taxonomy of avian Plasmodium parasites at subgenus level. It is preferable to limit use of the feature of absence or presence of globules in erythrocytic meronts to identification of natural infections at species level, on which the taxonomic validity of this feature also needs to be tested. Experimental sporozoite-induced infections of same parasites lineages possessing and not possessing globules in different avian hosts might help to answer the question about taxonomic value of this feature. Until additional information is available, Papernaia is considered as a synonym of subgenus Novyella.

\section{Conclusion}

Based on available morphological data and DNA sequence information, 55 species of avian Plasmodium parasites can be readily distinguished. Species of subgenus Novyella predominate among them. Dichotomous keys for identification of these parasites were compiled allowing identification of these pathogens using morphological features of their blood stages. The majority of described avian Plasmodium species are mainly transmitted in countries with warm climates. The obstacles for their global spread remain insufficiently understood, mainly because of limited information on life cycles and vectors of the majority of described parasites of tropical birds. The lists of synonymous names as well as names of the categories species inquirenda and incertae sedis should be considered in future taxonomic work of avian malaria parasite at species level. The majority of described Plasmodium parasites have not been characterized using molecular markers, which 

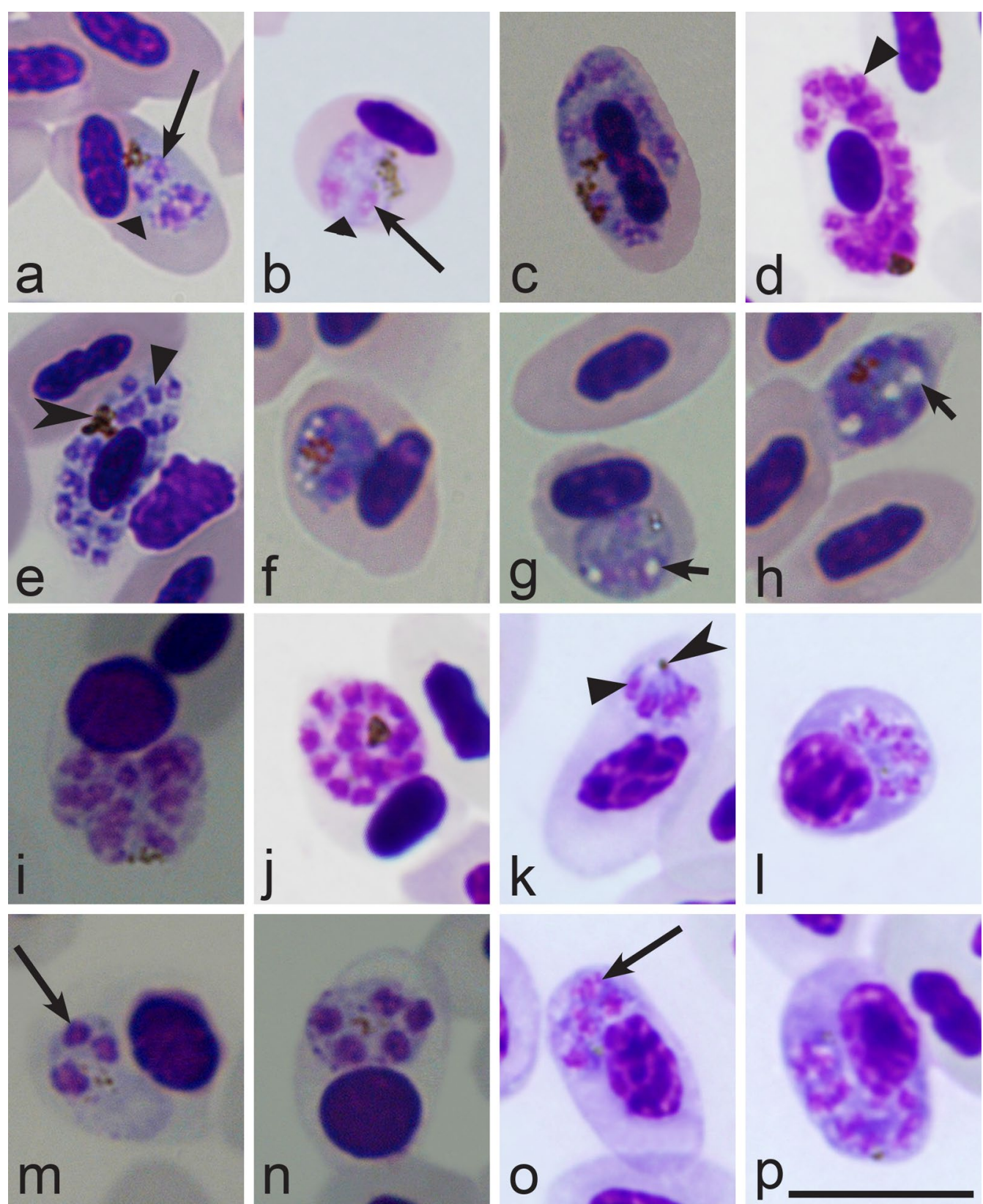

Fig. 2 Morphological features of erythrocytic meronts and their host cells of avian Plasmodium parasites, which are used for Haemamoeba, Giovannolaia and Huffia species identification. Growing $(\mathbf{a}-\mathbf{c}, \mathbf{f}-\mathbf{h}, \mathbf{I}-\mathbf{p})$ and mature $(\mathbf{d}, \mathbf{e}, \mathbf{i}-\mathbf{k})$ meronts at different stages of their development. Note presence of the plentiful cytoplasm and large nuclei in early growing meronts $(\mathbf{a}, \mathbf{b}, \mathbf{f}-\mathbf{h}, \mathbf{m}-\mathbf{p})$, marked vacuolization of the cytoplasm (f-h), elongate shape of mature merozoites $(\mathbf{k})$, presence of meronts in erythroblasts $(\mathbf{i}, \mathbf{I}-\mathbf{n})$ and other immature red blood cells $(\mathbf{k}, \mathbf{o}, \mathbf{p})$, and distinct smooth outline in growing erythrocytic meronts $(\mathbf{m}, \mathbf{n})$. Short simple arrows—vacuoles. Wide triangle arrowheads—the cytoplasm. Other symbols are as in Fig. 1. Explanations are given in the text 

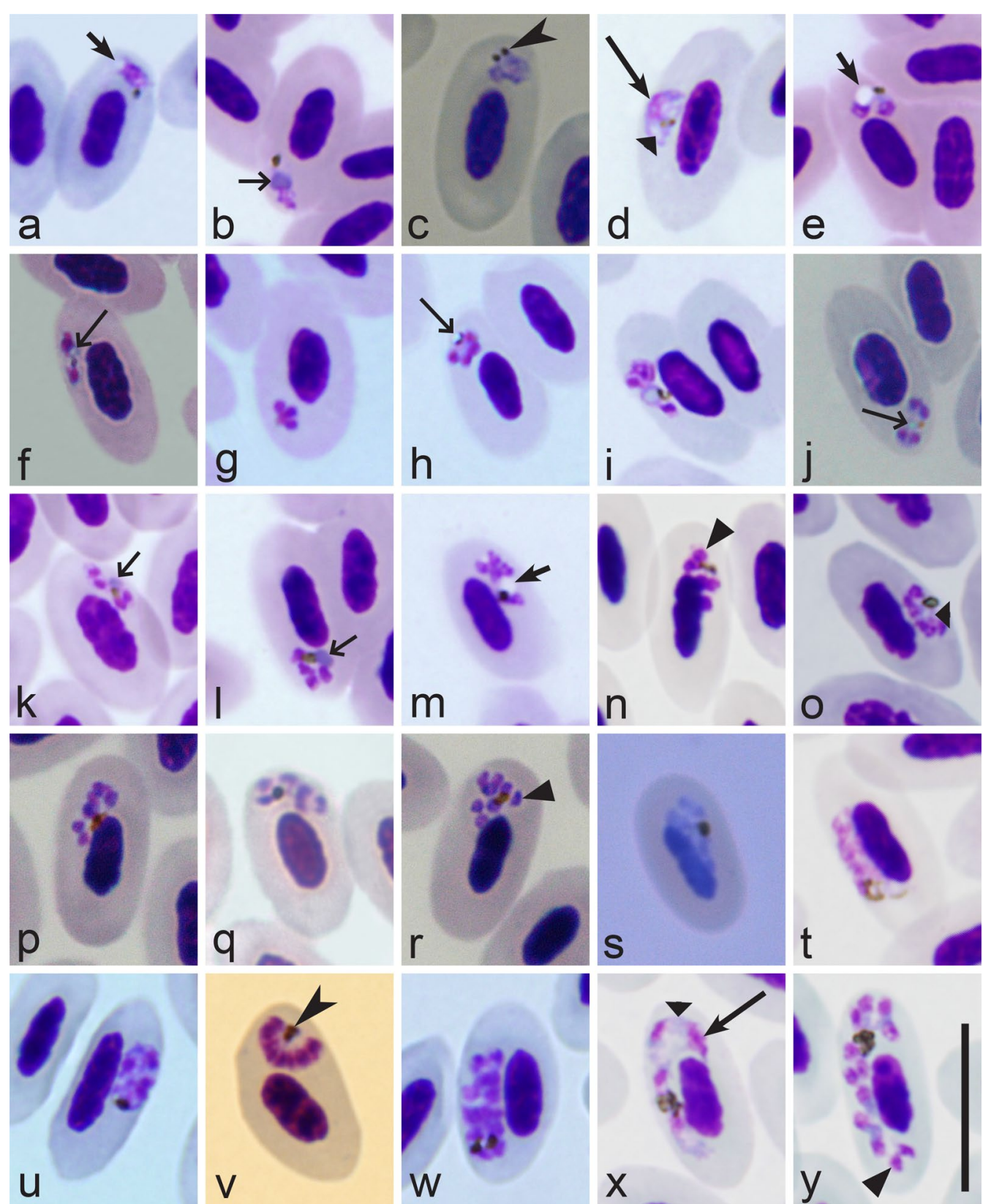

Fig. 3 Morphological features of erythrocytic meronts and their host cells of avian Plasmodium parasites, which are used for Novyella and Giovannolaia species identification. Trophozoites (a-d) and erythrocytic meronts (e-y) on different stages of maturation. Note presence of large vacuoles $(\mathbf{a}, \mathbf{e}, \mathbf{m})$, refractive small globules $(\mathbf{f}, \mathbf{h}$-j), bluish non-refractive globules $(\mathbf{b}, \mathbf{k}, \mathbf{l})$, fan-like mature meronts $(\mathbf{o}, \mathbf{v})$, strictly nucleopilic position $(\mathbf{n}, \mathbf{t})$, the scanty (nearly invisible) cytoplasm $(\mathbf{a}, \mathbf{b}, \mathbf{e}-\mathbf{I})$ and the prominent (readily visible) cytoplasm $(\mathbf{d}, \mathbf{x})$ in parasites on different stages of their development. Triangle wide long arrows - refractive globules. Triangle wide short arrows—bluish (non-refractive) globules. Other symbols are as in Fig. 1. Explanations are given in the text 

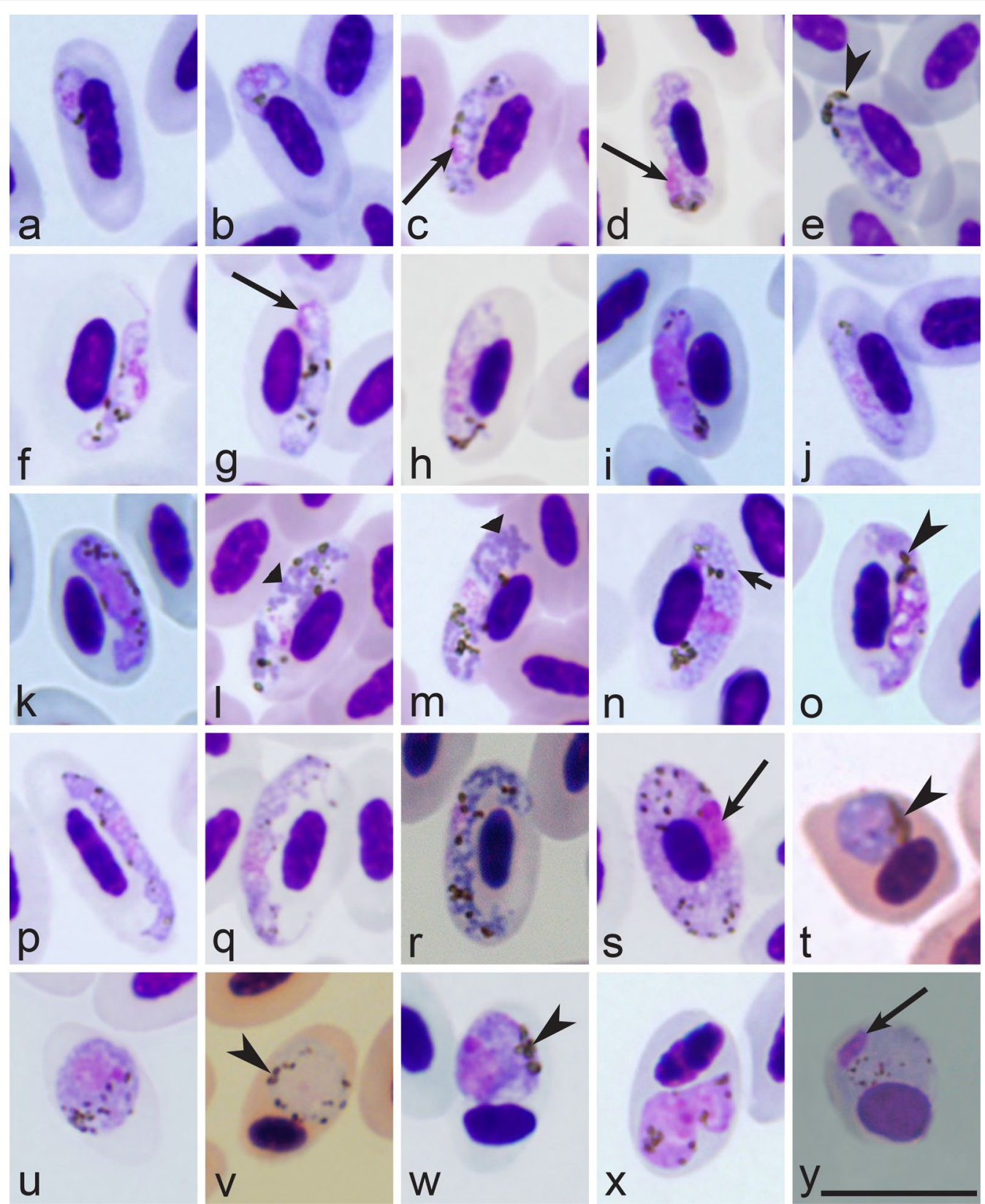

Fig. 4 Morphological features of gametocytes and their host cells of avian Plasmodium parasites, which are used for species identification Macrogametocytes $(\mathbf{a}-\mathbf{g}, \mathbf{k}-\mathbf{u}, \mathbf{w}-\mathbf{y})$ and microgametocytes $(\mathbf{h}-\mathbf{j}, \mathbf{v})$. Note long outgrowth $(\mathbf{f})$, terminal position of pigment granules $(\mathbf{e})$ and nucleus $(\mathbf{g})$, granular $(\mathbf{I}, \mathbf{m})$ and vacuolated $(\mathbf{n})$ appearance of the cytoplasm, slender $(\mathbf{p}-\mathbf{r})$ and circumnuclear (s) shapes of gametocytes, clumps of pigment granules located near the parasite margin $(\mathbf{t}, \mathbf{w})$, distinct smooth outline of nucleus $(\mathbf{y})$. Symbols as in Figs. 1, 2, 3. Explanations are given in the text 
a
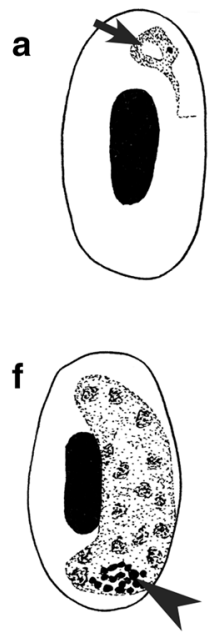

k
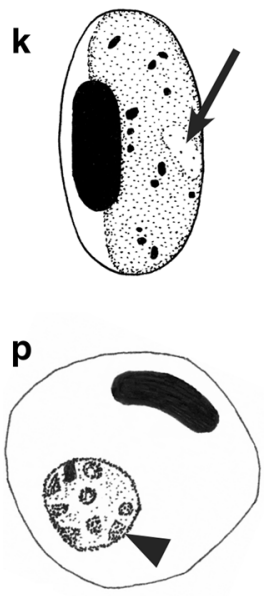

$\mathbf{u}$

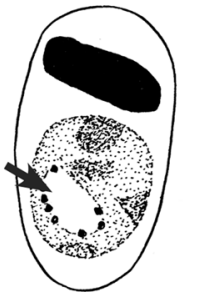

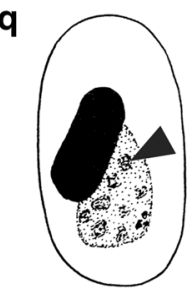

b

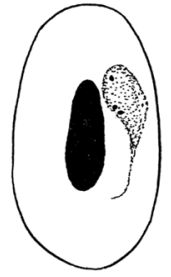

g

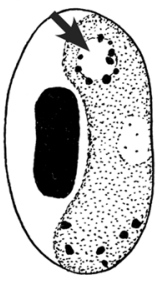

I
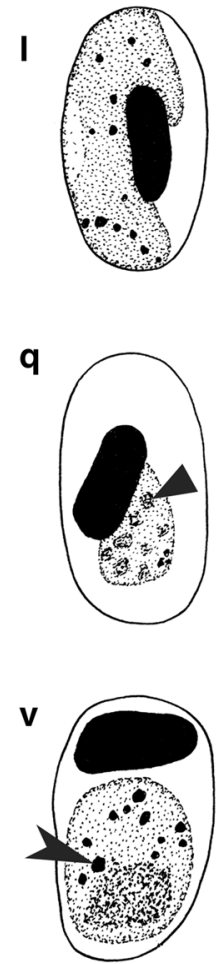

c
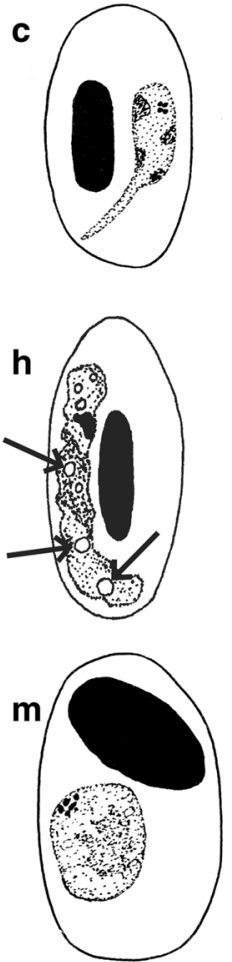

$r$

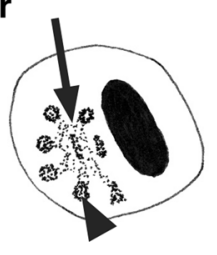

d

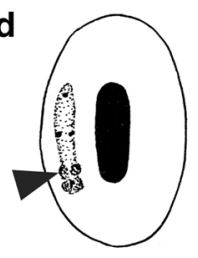

e

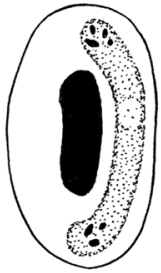

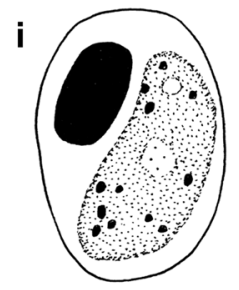

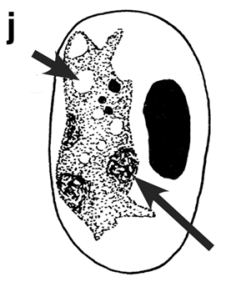

n

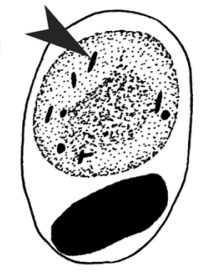

0

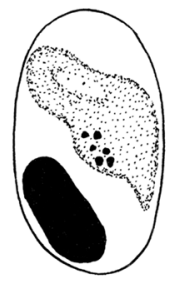

s

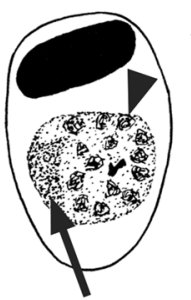

$\mathbf{t}$
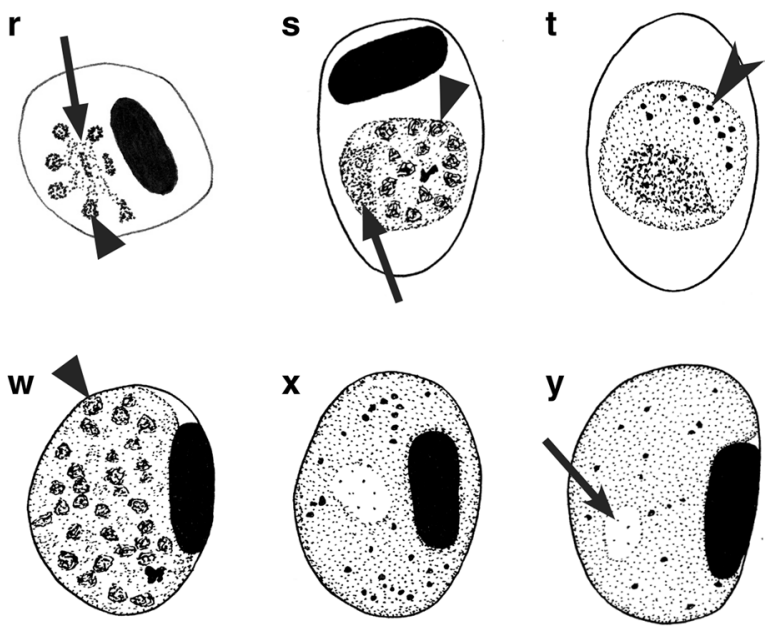

Fig. 5 Morphological features of blood stages and their host cells of avian Plasmodium parasites, which are used for species identification. Young trophozoite (a) and gametocyte $(\mathbf{b})$, growing erythrocytic meronts $(\mathbf{c}, \mathbf{d}, \mathbf{j}, \mathbf{u})$, mature erythrocytic meronts $(\mathbf{f}, \mathbf{p}-\mathbf{s}, \mathbf{w})$, and mature gametocytes $(\mathbf{e}$, $\mathbf{g}-\mathbf{i}, \mathbf{k}-\mathbf{o}, \mathbf{t}, \mathbf{v}, \mathbf{x}, \mathbf{y})$. Note presence of long outgrowths $(\mathbf{a}-\mathbf{c})$, terminal position of nuclei in meront (d), slender shape of gametocyte (e), aggregation of pigment granules at one end of gametocyte $(\mathbf{f})$, rod-like pigment granules $(\mathbf{n})$, large vacuoles $(\mathbf{g}, \mathbf{j}$, $\mathbf{u})$, refractive globules in gametocyte $(\mathbf{h})$, oblique position of gametocytes in erythrocytes $(\mathbf{i}, \mathbf{o})$, strictly nucleophilic erythrocytic meronts $(\mathbf{q})$, residual cytoplasm in erythrocytic meronts $(\mathbf{r}, \mathbf{s})$, rounded shape of infected erythrocytes $(\mathbf{p}, \mathbf{w}-\mathbf{y})$. Triangle long arrows-residual body in mature meront. Symbols as in Figs. 1, 2, 3, 4. Explanations are given in the text 

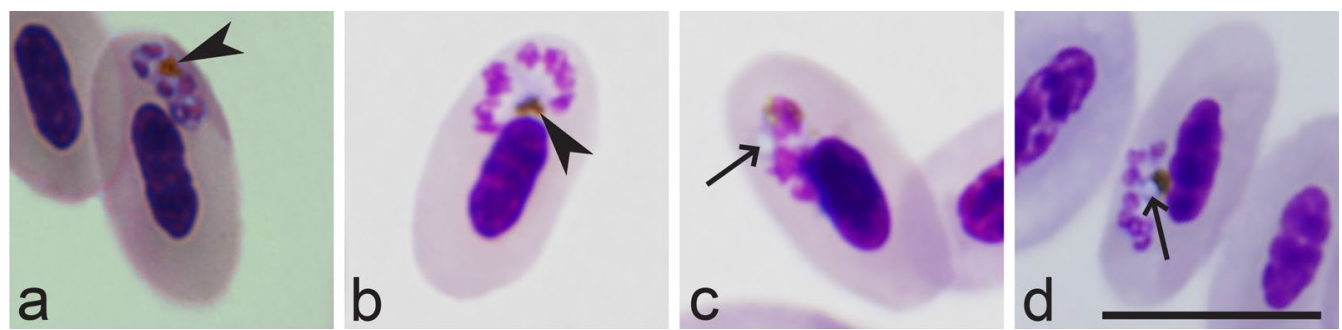

Fig. 6 Maturing erythrocytic meronts of Plasmodium ashfordi (lineage pGRW2) in naturally infected the Common cuckoo Cuculus canorus (a) and experimentally infected Eurasia siskin Carduelis spinus (b-d) during the first (b) and 3-4th (c, d) passages of infected blood. Note that refractive globules were absent in erythrocytic meronts during the natural infection (a) and the first passage of the experimental infection (b), but develop in subsequent passages of the same strain in Eurasian siskin. Symbols are as in Figs. 1 and 3

\section{development is an essential task for current avian malaria researchers.}

\section{Authors' contributions}

GV collected published articles and collection material, analysed the literature data and wrote the manuscript; GV and TAl analysed preparations of the blood stages; TAl and GV prepared plates of images. Both authors read and approved the final manuscript.

\section{Acknowledgements}

This article benefited from comments made by Richard W. Ashford and Carolina R. F. Chagas. We thank R. Adlard, E. Hoberg, A. Warren, I. Landau, C. Atkinson and N.E. Matta for assistance in accessing parasite material and D. Bukauskaitè, M. Ilgūnas, V. Palinauskas and R. Žiegytė, for participation in field work during collection of samples.

\section{Competing interests}

The authors declare that they have no competing interests.

\section{Availability of data and materials}

All data generated during this study are included in this published article.

\section{Consent for publication}

Not applicable.

\section{Ethics approval and consent to participate}

Not applicable.

\section{Funding}

This study was funded by the Research Council of Lithuania (No. MIP-045/2015).

\section{Publisher's Note}

Springer Nature remains neutral with regard to jurisdictional claims in published maps and institutional affiliations.

Received: 24 March 2018 Accepted: 15 May 2018

Published online: 29 May 2018

\section{References}

1. Garnham PCC. Malaria parasites and other Haemosporidia. Oxford: Blackwell; 1966.

2. Seed TM, Manwell RD. Plasmodia of birds. In: Kreier JP, editor. Parasitic protozoa, vol III. Gregarines, Haemogregarines, Coccidia, Plasmodia, and Haemoproteids. New York: Academic Press; 1977. p. 311-57.
3. Bennett GF, Whiteway M, Woodworth-Lynas C. A host-parasite catalogue of the avian haematozoa. Occasional Papers in Biology. St. John's: Memorial University of Newfoundland; 1982. p. 243.

4. Atkinson CT, Thomas NJ, Hunter DB. Parasitic diseases of wild birds. Oxford:Wiley-Blackwell; 2008.

5. Clark NJ, Clegg SM, Lima MR. A review of global diversity in avian haemosporidians (Plasmodium and Haemoproteus: Haemosporida): new insights from molecular data. Int J Parasitol. 2014;44:329-38.

6. Hellgren $\mathrm{O}$, Atkinson CT, Bensch S, Albayrak T, Dimitrov D, Ewen JG, et al. Global phylogeography of the avian malaria pathogen Plasmodium relictum based on MSP1 allelic diversity. Ecography. 2015;38:842-50.

7. Sehgal RN. Manifold habitat effects on the prevalence and diversity of avian blood parasites. Int J Parasitol Parasites Wildl. 2015:4:421-30.

8. Valkiūnas G. Avian malaria parasites and other Haemosporidia. Boca Raton: CRC; 2005

9. Santiago-Alarcon D, Palinauskas V, Schaefer HM. Diptera vectors of avian Haemosporidian parasites: untangling parasite life cycles and their taxonomy. Biol Rev Camb Philos Soc. 2012;87:928-64.

10. Njabo K, Cornel AJ, Sehgal RNM, Loiseau C, Buermann W, Harrigan RJ, et al. Coquillettidia (Culicidae, Diptera) mosquitoes are natural vectors of avian malaria in Africa. Malar J. 2009;8:193.

11. Ejiri H, Sato Y, Kim KS, Tsuda Y, Murata K, Saito K, et al. Blood meal identification and prevalence of avian malaria parasite in mosquitoes collected at Kushiro Wetland, a subarctic zone of Japan. J Med Entomol. 2011;48:904-8.

12. Garnham PCC. Malaria in its various vertebrate hosts. In: Kreier JP, editor. Malaria. Part 1. Epidemiology, chemotherapy, morphology and metabolism. New York: Academic Press; 1980. p. 95-144.

13. Sherman IW. Malaria: parasite biology, pathogenesis, and protection. Washington: ASM; 1998

14. Cowman AF, Healer J, Marapana D, Marsh K. Malaria: biology and disease. Cell. 2016;167:610-24.

15. Valkiūnas G, Žiegytè R, Palinauskas V, Bernotienè R, Bukauskaitè $D$ Ilgūnas M, et al. Complete sporogony of Plasmodium relictum (lineage pGRW4) in mosquitoes Culex pipiens pipiens, with implications on avian malaria epidemiology. Parasitol Res. 2015;144:3075-85.

16. Žiegytė R, Bernotienè R, Bukauskaitè D, Palinauskas V, lezhova TA, Valkiünas G. Complete sporogony of Plasmodium relictum (lineages pSGS1 and pGRW11) in mosquito Culex pipiens pipiens form molestus, with implications to avian malaria epidemiology. J Parasitol. 2014;100:878-82.

17. Howe L, Castro IC, Schoener ER, Hunter S, Barraclough RK, Alley MR. Malaria parasites (Plasmodium spp.) infecting introduced, native and endemic New Zealand birds. Parasitol Res. 2012;110:913-23.

18. Loiseau C, Harrigan RJ, Cornel AJ, Guers SL, Dodge M, Marzec T, et al. First evidence and predictions of Plasmodium transmission in Alaskan bird populations. PLoS ONE. 2012;7:e44729.

19. Schoener ER, Banda M, Howe L, Castro IC, Alley MR. Avian malaria in New Zealand. NZ Vet J. 2014;62:189-98.

20. Marzal A. Recent advances in studies on avian malaria parasites. In: Okwa OO, editor. Malaria parasites. In Tech: Rijeka; 2012. p. 135-58. 
21. Bensch S, Hellgren O, Pérez-Tris J. MalAvi: a public database of malaria parasites and related haemosporidians in avian hosts based on mitochondrial cytochrome b lineages. Mol Ecol Resour. 2009;9:1353-8.

22. Zehtindjiev P, Križanauskienè A, Scebba S, Dimitrov D, Valkiūnas G, Heggemann A, et al. Haemosporidian infections in skylarks (Alauda arvensis): a comparative PCR-based and microscopy study on the parasite diversity and prevalence in southern Italy and the Netherlands. Eur J Wildl Res. 2012;58:335-44.

23. Palinauskas $V$, Žiegytè $R$, lezhova TA, Ilgūnas $M$, Bernotienè $R$, Valkiūnas G. Description, molecular characterisation, diagnostics and life cycle of Plasmodium elongatum (lineage pERIRUB01), the virulent avian malaria parasite. Int J Parasitol. 2016;46:697-707.

24. Ilgūnas M, Bukauskaitè D, Palinauskas V, lezhova TA, Dinhopl N, Nedorost $\mathrm{N}$, et al. Mortality and pathology in birds due to Plasmodium (Giovannolaia) homocircumflexum infection, with emphasis on the exoerythrocytic development of avian malaria parasites. Malar J. 2016;15:256.

25. Valkiūnas G, lezhova TA. Exo-erythrocytic development of avian malaria and related haemosporidian parasites. Malar J. 2017;16:101.

26. Corradetti A, Garnham PCC, Neri L, Scanga M, Cavallini C. A redescription of Plasmodium (Haemamoeba) relictum (Grassi and Feletti, 1891). Parassitologia. 1970;12:1-10.

27. Bennett GF, Warren M, Cheong WH. Biology of the Malaysian strain of Plasmodium juxtanucleare Versiani and Gomes, 1941. II. The sporogonic stages in Culex (Culex) sitiens Wiedmann. J Parasitol. 1966;52:647-52.

28. Martinsen ES, Perkins SL, Schall JJ. A three-genome phylogeny of malaria parasites (Plasmodium and closely related genera): evolution of life-history traits and host switched. Mol Phylogenet Evol. 2008;47:261-73.

29. Outlaw DC, Ricklefs RE. Species limits in avian malaria parasites (Haemosporida): how to move forward in the molecular era. Parasitology. 2014;141:1223-32.

30. Perkins SL. Malaria's many mates: past, present and future of the systematics of the order Haemosporida. J Parasitol. 2014;100:11-25.

31. Outlaw RK, Counterman B, Outlaw DC. Differential patterns of molecular evolution among Haemosporidian parasite groups. Parasitology. 2015;142:612-22.

32. Bensch S, Canbäck B, DeBarry JD, Johansson T, Hellgren O, Kissinger JC, et al. The genome of Haemoproteus tartakovskyi and its relationship to human malaria parasites. Genome Biol Evol. 2016:8:1361-73.

33. Pacheco AM, Matta NE, Valkiūnas G, Parker PG, Mello B, Stanley CE $J$ r, et al. Mode and rate of evolution of haemosporidian mitochondrial genomes: timing the radiation of avian parasites. Mol Biol Evol. 2018:35:383-403.

34. Adams Y, Kuhnrae P, Higgins MK, Ghumra A, Rowe JA. Rosetting Plasmodium falciparum-infected erythrocytes bind to human brain microvascular endothelial cells in vitro, demonstrating a dual adhesion phenotype mediated by distinct $P$. falciparum erythrocyte membrane protein 1 domains. Infect Immun. 2014;82:949-59.

35. Palinauskas V, Žiegytè R, Ilgūnas M, lezhova TA, Bernotienè R, Bolshakov $C$, et al. Description of the first cryptic avian malaria parasite, Plasmodium homocircumflexum n. sp., with experimental data on its virulence and development in avian hosts and mosquitoes. Int J Parasitol. 2015;45:51-62.

36. Huchzermeyer FW, van der Vyver FH. Isolation of Plasmodium circumflexum from wild guineafowl (Numida meleagris) and the experimental infection in domestic poultry. Avian Pathol. 1991;20:213-23.

37. Huchzermeyer FW. Pathogenicity and chemotherapy of Plasmodium durae in experimentally infected domestic turkeys. Onderstepoort J Vet Res. 1993;60:103-10.

38. Palinauskas V, Valkiūnas G, Bolshakov CV, Bensch S. Plasmodium relictum (lineage P-SGS1): effects on experimentally infected passerine birds. Exp Parasitol. 2008;120:372-80.

39. Vanstreels RE, Kolesnikovas CK, Sandri S, Silveira P, Belo NO, Ferreira Junior FC, et al. Outbreak of avian malaria associated to multiple species of Plasmodium in magellanic penguins undergoing rehabilitation in southern Brazil. PLoS ONE. 2014;9:e94994.

40. Dimitrov D, Palinauskas V, lezhova TA, Bernotienè R, Ilgūnas $M$, Bukauskaitė D, et al. Plasmodium spp.: an experimental study on vertebrate host susceptibility to avian malaria. Exp Parasitol. 2015;148:1-16.
41. Vanstreels RE, da Silva-Filho RP, Kolesnikovas CK, Bhering RC, Ruoppolo $\checkmark$, Epiphanio $S$, et al. Epidemiology and pathology of avian malaria in penguins undergoing rehabilitation in Brazil. Vet Res. 2015;46:30.

42. Valkiūnas G, Ashford RW. Natural host range is not a valid taxonomic character. Trends Parasitol. 2002;18:528-9.

43. Kim KS, Tsuda Y, Yamada A. Blood meal identification and detection of avian malaria parasite from mosquitoes (Diptera: Culicidae) inhabiting coastal areas of Tokyo Bay, Japan. J Med Entomol. 2009;46:1230-4.

44. Dinhopl N, Nedorost N, Mostegl MM, Weissenbacher-Lang C, Weissenböck H. In situ hybridization and sequence analysis reveal an association of Plasmodium spp. with mortalities in wild passerine birds in Austria. Parasitol Res. 2015:114:1455-62.

45. Valkiūnas G, lezhova TA, Shapoval AP. High prevalence of blood parasites in hawfinch Coccothraustes coccothraustes. J Nat Hist. 2003;37:2647-52.

46. Valkiūnas $G$, Bensch S, lezhova TA, Križanauskienè A, Hellgren O, Bolshakov CV. Nested cytochrome $b$ polymerase chain reaction diagnostics underestimate mixed infections of avian blood haemosporidian parasites: microscopy is still essential. J Parasitol. 2006;92:418-22.

47. Martínez J, Martínez-De La Puente J, Herrero J, Del Cerro S, Lobato E, Rivero-De Aguilar J, Cerro S, Lobato E, Rivero-De Aguilar J, et al. A restriction site to differentiate Plasmodium and Haemoproteus infections in birds: on the inefficiency of general primers for detection of mixed infections. Parasitology. 2009;136:713-22.

48. Bernotienè R, Palinauskas V, lezhova TA, Murauskaitè D, Valkiūnas G. Avian haemosporidian parasites (Haemosporida): a comparative analysis of different polymerase chain reaction assays in detection of mixed infections. Exp Parasitol. 2016;163:31-7.

49. Mantilla JS, González AD, Valkiūnas G, Moncada LI, Matta NE. Description and molecular characterization of Plasmodium (Novyella) unalis sp. nov. from the Great Thrush (Turdus fuscater) in highland of Colombia. Parasitol Res. 2013;112:4193-204.

50. Silveira P, Belo NO, Lacorte GA, Kolesnikovas CKM, Vanstreels RET, Steindel $M$, et al. Parasitological and new molecular-phylogenetic characterization of the malaria parasite Plasmodium tejerai in South American penguins. Parasitol Int. 2013:62:165-71.

51. Savage AF, Ariey F, Greiner EC. A new species of Plasmodium from Malagasy vangas. J Parasitol. 2005;9:926-30.

52. Valkiūnas G, lezhovaTA, Loiseau C, Chasar A, SmithTB, Sehgal RNM. New species of haemosporidian parasites (Haemosporida) from African rainforest birds, with remarks on their classification. Parasitol Res. 2008;103:1213-28.

53. Paperna I, Yosef R, Landau I. Plasmodium spp. in raptors on the EurasianAfrican migration route. Parasite. 2007;14:313-22.

54. Paperna I, Yosef R, Chavatte JM, Grill H, Landau I. Species of Plasmodium of passerine birds with four nuclei, with description of new species. Acta Parasitol. 2008;2008(53):227-36.

55. Valkiūnas G, lezhova TA, Loiseau C, Smith TB, Sehgal RNM. New malaria parasites of the subgenus Novyella in African rainforest birds, with remarks on their high prevalence, classification and diagnostics. Parasitol Res. 2009;104:1061-77.

56. Chavatte JM, Chiron F, Chabaud A, Landau I. Probable speciations by "host-vector 'fidelity"': 14 species of Plasmodium from magpies. Parasite. 2007;14:21-37 (in French).

57. Chavatte JM, Grés V, Snounou G, Chabaud A, Landau I. Plasmodium (Apicomplexa) of the skylark (Alauda arvensis). Zoosystema. 2009:31:369-83.

58. Walther EL, Valkiūnas G, González AD, Matta NE, Ricklefs RE, Cornel A, et al. Description, molecular characterization, and patterns of distribution of a widespread New World avian malaria parasite (Haemosporida: Plasmodiidae), Plasmodium (Novyella) homopolare sp. nov. Parasitol Res. 2014;113:3319-32.

59. Zehtindjiev P, Križanauskienè A, Bensch S, Palinauskas V, Asghar M, Dimitrov $D$, et al. A new morphologically distinct avian malaria parasite that fails detection by established PCR-based protocols for amplification of the cytochrome b gene. J Parasitol. 2012;98:657-65.

60. Telford SR. The hemoparasites of the reptilian. Boca Raton: CRC; 2009.

61. Lainson R. Atlas of protozoan parasites of the Amazonian fauna of Brazil. Haemosporida of reptiles, vol. 1. Ananindeua: Instituto Evandro Chagas; 2012.

62. Gabaldon A, Ulloa G, Zerpa N. Fallisia (Plasmodioides) neotropicalis subgen. nov. sp. nov. from Venezuela. Parasitology. 1985;90:217-25. 
63. Campbell TW. Avian hematology and cytology. Ames: lowa State University Press; 1995.

64. Valkiūnas $G$, lezhova TA, Križanauskienė A, Palinauskas V, Sehgal RNM, Bensch S. A comparative analysis of microscopy and PCR-based detection methods for blood parasites. J Parasitol. 2008;94:1395-401.

65. Landau I, Chabaud AG, Bertani S, Snounou G. Taxonomic status and redescription of Plasmodium relictum (Grassi et Feletti, 1891), Plasmodium maior Raffaele, 1931, and description of P. bigueti n. sp. in sparrows. Parassitologia. 2003:45:119-23.

66. Valkiūnas $G$, Ilgūnas M, Bukauskaitè D, Palinauskas V, Bernotienè R, lezhova TA. Molecular characterization and distribution of Plasmodium matutinum, a common avian malaria parasite. Parasitology. 2017; 144:1726-35.

67. Palinauskas V, Kosarev V, Shapoval A, Bensch S, Valkiūnas G. Comparison of mitochondrial cytochrome $b$ lineages and morphospecies of two avian malaria parasites of the subgenera Haemamoeba and Giovannolaia (Haemosporida: Plasmodiidae). Zootaxa. 2007;1626:39-50.

68. Marzal A, Ricklefs RE, Valkiūnas G, Albayrak T, Arriero E, Bonneaud C, et al. Diversity, loss and gain of malariae parasites in a globally invasive bird. PLoS ONE. 2011;6:e21905.

69. Beadell JS, Ishtiaq F, Covas R, Melo M, Waren BH, Atkinson CT, et al. Global phylogeographic limits of Hawaii's avian malaria. Proc R Soc Lond B Biol Sci. 2006;273:2935-44.

70. Bueno MG, Lopez RP, de Menezes RM, de Costa-Nascimento MJ, Lima GF, Araújo RA, et al. Identification of Plasmodium relictum causing mortality in penguins (Spheniscus magellanicus) from São Paulo Zoo, Brazil. Vet Parasitol. 2010;173:123-7.

71. Valkiūnas G, Zehtindjiev P, Hellgren O, Ilieva M, lezhova TA, Bensch S. Linkage between mitochondrial cytochrome b lineages and morphospecies of two avian malaria parasites, with a description of Plasmodium (Novyella) ashfordi sp. nov. Parasitol Res. 2007;100:1311-22.

72. Valkiūnas G, Ilgūnas M, Bukauskaitė D, Žiegytè R, Bernotienė R, Jusys $\mathrm{V}$, et al. Plasmodium delichoni $\mathrm{n}$. sp.: description, molecular characterisation and remarks on the exoerythrocytic merogony, persistence, vectors and transmission. Parasitol Res. 2016;115:2625-36.

73. Ricklefs RE, Soares L, Ellis VA, Latta SC. Avian migration and the distribution of malaria parasites in New World passerine birds. J Biogeogr. 2017:44:1113-23.

74. Zehtindjiev P, Ilieva M, Westerdahl H, Hansson B, Valkiūnas G, Bensch S. Dynamics of parasitemia of malaria parasites in a naturally and experimentally infected migratory songbird, the great reed warbler Acrocephalus arundinaceus. Exp Parasitol. 2008;119:99-110.

75. Palinauskas V, Valkiūnas G, Bolshakov CV, Bensch S. Plasmodium relictum (lineage SGS1) and Plasmodium ashfordi (lineage GRW2): the effects of the co-infection on experimentally infected passerine birds. Exp Parasitol. 2011;127:527-33.

76. Escalante AA, Freeland DE, Collins WE, Lal AA. The evolution of primate malaria parasites based on the gene encoding cytochrome $b$ from the linear mitochondrial genome. Proc Natl Acad Sci USA. 1998;95:8124-9.

77. Bensch S, Stjernman M, Hasselquist D, Östman Ö, Hansson B, Westerdahl $\mathrm{H}$, et al. Host specificity in avian blood parasites: a study of Plasmodium and Haemoproteus mitochondrial DNA amplified from birds. Proc Biol Sci. 2000:276:1583-9.

78. International Commission on Zoological Nomenclature. International code of zoological nomenclature. 4th ed. London: The International Trust for Zoological Nomenclature; 1999.

79. Landau I, Chavatte JM, Peters W, Chabaud A. The sub-genera of avian Plasmodium. Parasite. 2010;17:3-7.

80. Chavatte JM, Uzbekov R, Paperna I, Richard-Lenoble D, Landau I. Ultrastructure of erythrocytic stages of avian Plasmodium spp. of the sub-genus Novyella and its "globule". Parasite. 2010;17:123-7.

81. Versiani V, Gomes BF. Sobre um novo hematozoário da galinha"Plasmodium juxtanucleare" n. sp. (Nota prévia). Rev Brasil Biol. 1941;1:231-3.

82. Raffaele G II. Plasmodium della civetta (Athene noctua). Riv Malariol. 1931;10:684-8.

83. Brumpt E. Paludisme aviaire: Plasmodium gallinaceum n. sp. de la poule domestique. C R Hebd Séances Acad Sci. 1935;200:783-5

84. Bano L, Abbasi Z. A new species of avian malaria parasite, Plasmodium coturnixi, from Coturnix coturnix from Kohat (N.W.F.P., Pakistan). Bull Zool. 1983;1:17-22.
85. Grassi B, Feletti R. Malariaparasiten in den Vögeln. Centralbl Bakteriol Parasitenkd. 1891;9:403-9, 429-33, 461-7.

86. Hartman E. Three species of bird malaria, Plasmodium praecox, P. cathemerium n. sp. and P. inconstans n. sp. Arch Protistenkd. 1927;60:1-7.

87. Corradetti A, Verolini F, Neri I. Plasmodium (Haemamoeba) giovannolai $\mathrm{n}$. sp. parassita di Turdus merula. Parassitologia. 1963;5:11-8.

88. Huff CG. A new variety of Plasmodium relictum from the robin. J Parasitol. 1937;23:400-4.

89. Gabaldon A, Ulloa G. Plasmodium (Haemamoeba) tejerai sp. n. del pavo domésstico (Meleagris gallopavo) de Venezuela. Bol Dir Malariol San Amb. 1977;17:255-73.

90. Lucena D. Malária aviária I_Plasmodium lutzi n. sp. Parasita da Saracura (Aramides cajanea cajanea, Müller). Bull Biol (São Paulo). 1939:4:27-31.

91. Mantilla JS, Matta NE, Pacheco MA, Escalante AA, Gonzalez AD, Moncada LI. Identification of Plasmodium (Haemamoeba) lutzi (Lucena, 1939) from Turdus fuscater (Great Thrush) in Colombia. J Parasitol. 2013;99:662-8.

92. Schwetz J. Sur un Plasmodium aviaire à formes de division allongées, Plasmodium fallax, n. sp. Arch Inst Pasteur Alger. 1930;8:289-96.

93. Manwell RD, Kuntz RE. Plasmodium hegneri n. sp. from the European Teal Anas c. crecca in Taiwan. J Protozool. 1966;13:437-40.

94. Huang J-C. A new species of the genus Plasmodium_Plasmodium leanucleus (Eucoccidia: Plasmodiidae). Acta Vet Zoot Sinica. 1991;16:257-62

95. Bray RS. On the parasitic Protozoa of Liberia. VII-Haemosporidia of owls. Arch Inst Pasteur Alger. 1962;40:201-7.

96. Manwell RD. Plasmodium octamerium n. sp., an avian malaria parasite from the pintail whydah bird Vidua macroura. J Protozool. 1968;15:680-5.

97. Kikuth W. Immunbiologische und chemotherapeutische Studien an verschiedenen Stämmen von Vogelmalaria. Zentralbl Bakteriol Parasitenkd Infektionskr Hyg I Abt Orig. 1931;121:401-9.

98. Coggeshall LT. Plasmodium lophurae, a new species of malaria parasite pathogenic for the domestic fowl. Am J Hyg. 1938;27:615-8.

99. Garnham PCC. A new malaria parasite of pigeons and ducks from Venezuela. Protistologica. 1977;13:113-25.

100. Manwell RD. How many species of avian malaria parasites are there? J Parasitol. 1934;20:334.

101. Muniz J, de Soares RRL. Nota sôbre um parasita do gênero Plasmodium encontrado no Ramphastos toco Müller, 1776, "Tugano-Açu", e diferente do Plasmodium huff: Plasmodium pinottii n. sp. Rev Bras Malariol. 1954:6:611-7.

102. Guindy $\mathrm{E}$, Hoogstraal H, Mohammed AHH. Plasmodium garnhami sp. nov. from the Egyptian hoopoe (Upupa epops major Brehm). Trans R Soc Trop Med Hyg. 1965;59:280-4

103. Manwell RD. A new species of avian Plasmodium. J Protozool. 1962:9:401-3.

104. Herman CM. Plasmodium durae, a new species of malaria parasite from the common turkey. Am J Hyg. 1941;34:22-6.

105. Shillinger JE. Diseases of wildlife and their relationship to domestic livestock. Washington: USDA Yearbook of Agriculture; 1942. p. 1217-25.

106. Stabler RM, Kitzmiller NJ, Braun CE. Plasmodium in a Darwin's tinamou from Colorado. J Parasitol. 1973.59.395.

107. Huff CG. Plasmodium hexamerium, n. sp. from the blue-bird, inoculable to canaries. Am J Hyg. 1935:22:274-7.

108. Novy FG, MacNeal WJ. Trypanosomes and bird malaria. Am Med. 1904:8:932-4

109. Telford SR, Nayar JK, Foster GW, Knight JW. Plasmodium forresteri n. sp., from raptors in Florida and Southern Georgia: its distinction from Plasmodium elongatum morphologically within and among host species and by vector susceptibility. J Parasitol. 1997;83:932-7.

110. Gabaldon A, Ulloa G. A new species of the subgenus Novyella (Haemosporina, Plasmodiidae) from Aramides cajanea (Gruiformes, Rallidae). In: Canning EU, editor. Parasitological topics. A presentation volume to P.C.C. Garnham, F.R.S. on the occasion of his 80th birthday. Madison: Society of protozoologists; 1981. p. 100-5.

111. Sergent E, Sergent E, Catanei A. Sur un parasite nouveau du paludisme des oiseaux. C R Hebd Séances Acad Sci Paris. 1928;186:809-11.

112. Manwell RD. How many species of avian malaria parasites are there? Am J Trop Med. 1935;15:265-83. 
113. Manwell RD, Sessler GJ. Plasmodium paranucleophilum n. sp. from a South American tanager. J Protozool. 1971;18:629-32.

114. Chagas CRF, Valkiūnas G, Nery CVC, Henrique PC, Gonzalez IHL, Monteiro EF, et al. Plasmodium (Novyella) nucleophilum from an Egyptian Goose in São Paulo Zoo, Brazil: microscopic confirmation and molecular characterization. Int J Parasitol Parasites Wildl. 2013;2:286-91.

115. Christensen BM, Barnes HJ, Rowley WA. Vertebrate host specificity and experimental vectors of Plasmodium (Novyella) kempi sp. n. from the eastern wild turkey in lowa. J Wildl Dis. 1983;19:204-13.

116. Carini A. Sur un nouvel hématozoaire du pigeon. C R Hebd Séances Mém Soc Biol. 1912;73:396-8.

117. Ilgūnas M, Palinauskas V, lezhova TA, Valkiūnas G. Molecular and morphological characterization of two avian malaria parasites (Haemosporida: Plasmodiidae), with description of Plasmodium homonucleophilum n. sp. Zootaxa. 2013;3666:49

118. de Jong AC. Plasmodium dissanaikei n. sp. a new avian malaria parasite from the rose-ringed parakeet of Ceylon, Psittacula krameri manillensis. Ceylon J Med Sci. 1971;20:41-5.

119. Huff CG. Plasmodium elongatum n. sp., an avian malarial organism with an elongate gametocyte. Am J Hyg. 1930;11:385-91.

120. Valkiūnas G, Zehtindjiev P, Dimitrov D, Križanauskienè A, lezhova TA, Bensch S. Polymerase chain reaction-based identification of Plasmodium (Huffia) elongatum, with remarks on species identity of haemosporidian lineages deposited in GenBank. Parasitol Res. 2008;102:1185-93.

121. Telford SR, Forrester DJ. Plasmodium (Huffia) hermani sp. n. from wild turkeys (Meleagris gallopavo) in Florida. J Protozool. 1975;22:324-8.

122. Muniz J, Soares R, Batista S. Sôbre uma espécie de Plasmodium parasita do Ramphastos toco Müller, 1776. Plasmodium huffi n. sp. Rev Bras Malariol. 1951;3:339-44

123. Wiersch SC, Maier WA, Kampen H. Plasmodium (Haemamoeba) cathemerium gene sequences for phylogenetic analysis of malaria parasites. Parasitol Res. 2005:96:90-4.

124. Perkins SL, Schall JJ. A molecular phylogeny of malarial parasites recovered from cytochrome b gene sequences. J Parasitol. 2002;88:972-8.

125. Omori S, Sato Y, Isobe T, Yukawa M, Murata K. Complete nucleotide sequences of the mitochondrial genomes of two avian malaria protozoa, Plasmodium gallinaceum and Plasmodium juxtanucleare. Parasitol Res. 2007;100:661-4

126. Celli A, Sanfelice F. Ueber die Parasiten des rothen Blutkörperchens im Menschen und in Thieren. Fortschr Med. 1891;9:499-511, 541-52, 581-6.

127. Gilruth JA, Sweet G, Dodd S. Notes on blood parasites. Proc R Soc Victoria. 1910;23:231-41.

128. Russell PF. Avian malaria studies, V. Plasmodium capistrani sp. nov., an avian malaria parasite in the Philippines. Philipp J Sci. 1932;48:269-89.

129. de Mello IF. Further contribution to the study of blood parasites of the Indian birds, together with a list of the hemoparasites hitherto recorded. J R Asiatic Soc Beng. 1936:2:95-122.

130. de Mello IF. A contribution to the study of the blood parasites of some Indian birds. Proc Indian Acad Sci (Sec. B). 1935:1:349-58.

131. Basu BC. Studies on a malarial infection in a paddy bird. J Malar Inst India. 1938;1:273-84.

132. Ishiguro H. Plasmodium japonicum, a new species of malaria parasite pathogenic for the domestic fowl. Bull Fac Agr Yamaguti Univ. 1957:8:723-33 (article in Japanese)

133. Raffaele G. Considerazioni sulla specificità dei plasmodili. Arch Zool Ital. 1966:51:273-83.

134. Labbé A. Recherches zoologiques et biologiques sur les parasites endoglobulaires du sang des vertébrés. Arch Zool Exp Gen. 1894:2:55-258.

135. Raffaele G. Osservazioni sui plasmodidi degli uccelli. Riv Malariol. 1930;9:209-18.

136. Corradetti A, Scanga M. Plasmodium (Novyella) vaughani subsp. merulae, n. subsp., parassita di Turdus merula, con descrizione del ciclo pre-eritrocitico. Parassitologia. 1972;14:85-93.

137. Das Gupta BM, Siddons LB. On a Plasmodium sp. of the Malay chestnutbellied munia [Munia atricapilla atricapilla (Vieill)]. Indian Med Gaz. $1941 ; 76: 148-50$

138. Laveran A. Sur une Haemamoeba d'une mésange (Parus major). C R Séances Soc Biol Fil. 1902:54:1121-4
139. Wolfson F Plasmodium oti n. sp. a Plasmodium from the eastern screech owl (Otus asio naevius), infective to canaries. Am J Hyg. 1936;24:94-101.

140. Johnston HT, Cleland JB. Notes on some parasitic Protozoa. Proc Linn Soc NSW. 1909;34:501-13

141. Brumpt E. Paludisme aviaire: Plasmodium paddae n. sp. du calfat (Padda oryzivora). Utilisation de ce parasite pour les recherches chimiothérapiques du paludisme. C R Hebd Séances Acad Sci. 1935:200:967-70.

142. Chakravarty M, Kar AB. Studies on Haemosporidia from Indian birdsSeries II. Proc Indian Acad Sci. 1945:22:63-9.

143. Fantham HB, Porter A. On a Plasmodium (Plasmodium relictum var. spheniscidae n. var.) observed in four species of penguins. Proc Zool Soc London. 1944:114:279-92.

144. Mazza S, Fiora A. Proteosoma de mirlo, Planesticus anthracinus (Burm.) y Leucocytozoon (sic) di Benteveo, Pitangus sulphuratus bolivianus (Latv.) y fueguero Piranga flava (Viell.) de Tumbaya, Jujuy. 5th Reunion Soc Argent Patol Reg Norte. 1930;2:993-5.

145. Laveran A, Marullaz M. Sur deux hémamibes et un toxoplasme du Liothrix luteus. Bull Soc Pathol Exot. 1914;7:21-5.

146. Brumpt E. Précis de Parasitologie. Paris: Masson; 1910.

147. Huang J-C, Huang D-F, Jiang J-B. A new species of the genus Plasmodium, Plasmodium arachnidi from the domestic pigeon in Guangzhou. Acta Vet Zoot Sinica. 1995:26:352-7.

148. Huang JC, Huang DF. A new species of the bird malarial parasite-Plasmodium (Novyella) bambusicolai (Sporozoa: Plasmodiidae). Acta Zoot Sinica. 1995:20:385-90.

149. Grés V, Les Landau I, de Lophura Plasmodium. Les Plasmodium de Lophura (Phasianidae): redescription de P. lophurae Coggeshall, 1938 et description de deux nouvelles espèces. Zoosystema. 1938:1997(19):545-55.

150. Sarkar AC, Ray HN. A new malarial parasite, Plasmodium (Garnhamella) conturnixae n. subgen., n. sp., from black breasted quail, Coturnix coromandelica (Aves: Galliformes). In: Progress in protozoology. Abstr. III Inter. Congr. Protozool. Leningrad; 1969. p. 353.

151. Laird M. Avian malaria in the Asian tropical subregion. Singapore: Springer; 1998

152. Grassi B, Feletti R. Parasites malariques chez les oiseaux. Arch Ital Biol. 1890;13:297-300

153. Castellani A, Chalmers AJ. Manual of tropical medicine. London: Manual of tropical Medicine; 1910

154. Stabler RM, Holt PA, Ellison LN. A new malaria from the spruce grouse. Colo-Wyo Acad Sci. 1965;5:49.

155. He J-G, Huang J-C. A new species of avian malaria parasite from Pycnonotus jocosus (Sporozoa: Plasmodiidae). Acta Zoot Sinica. 1993;18:129-33.

156. Oliger IM. New species of parasites of tetraonid birds. Uch zap Chuvashskogo gos ped inst. 1956;3:329-35 (in Russian).

157. Corradetti A, Neri I, Palmieri C, Verolini F, Giuliani V, Scanga M. Note su Plasmodium vaughani e su un plasmodio con ciclo schizogonico endoemoblastico di tipo elongatum rinvenuti in Turdus merula. Parassitologia. 1961;3:97-100

158. Partsvanidze MI. Plasmodium malariae raupachi from the turkey, new species. Zakavk vet vestn. 1914;6:86-7 (in Russian)

159. Stabler RM, Datel RJ. First record of malaria in American falcons (Falco sparverius). J Colo-Wyo Acad Sci. 1959;4:59.

160. Paperna I, Keong MSC, May CYA. Haematozoan parasites found in birds in peninsular Malaysia, Singapore, Sarawak and Jawa. Raffles Bull Zool. 2008:56:211-43.

161. Bray RS. A check-list of the parasitic Protozoa of West Africa with some notes on their classification. Bull Inst Fr Afr Noir. 1964;26:238-315.

162. Yarrington JT, Whitehair CK, Corwin RM. Vitamin E-selenium deficiency and its influence on avian malarial infection in the duck. J Nutr. 1973:103:231-41.

163. Fantham HB, Porter A. Plasmodium struthionis, sp. n., from Sudanese ostriches and Sarcocystis salvelini, sp. n., from Canadian speckled trout (Salvelinus fontinalis), together with a record of a Sarcocystis in the eel pout (Zoarces angularis). Proc Zool Soc London. 1943;1 12:25-30.

164. Bhaskar Rao TS, Devi A, Bhaskar Rao T. Studies on experimental infection of Plasmodium venkataramiahii of the crow Corvus splendens in the chicks. In: Abstracts. 5th Int Congr Protozool. New York. Abstr. 194; 1977. 